\title{
FLOWS OF MEASURES GENERATED BY VECTOR FIELDS
}

\author{
EMANUELE PAOLINI AND EUGENE STEPANOV
}

\begin{abstract}
The scope of the paper is twofold. We show that for a large class of measurable vector fields in the sense of N. Weaver (i.e. derivations over the algebra of Lipschitz functions), called in the paper laminated, the notion of integral curves may be naturally defined and characterized (when appropriate) by an ODE. We further show, that for such vector fields the notion of a flow of the given positive Borel measure similar to the classical one generated by a smooth vector field (in a space with smooth structure) may be defined in a reasonable way, so that the measure "flows along" the appropriately understood integral curves of the given vector field and the classical continuity equation is satisfied in the weak sense.
\end{abstract}

\section{INTRODUCTION}

Every sufficiently smooth and bounded vector field $V$ in the Euclidean space $E=\mathbb{R}^{n}$ is well-known to generate a canonical flow of a given finite Borel measure $\mu$ in $E$ by setting $\mu_{t}:=\varphi_{V \#}^{t} \mu, t \in \mathbb{R}^{+}$, where $\varphi_{V}^{t}(x):=\theta(t), \theta$ standing for the unique solution to the differential equation

$$
\dot{\theta}(t)=V(\theta(t))
$$

satisfying the initial condition $\theta(0)=x$. Such a flow satisfies the continuity equation

$$
\frac{\partial \mu_{t}}{\partial t}+\operatorname{div} v_{t} \mu_{t}=0
$$

in the weak sense in $E \times \mathbb{R}^{+}$, where $v_{t}: E \rightarrow E$ is some velocity field (of course, non unique for the given $V$, but in particular, this equation is satisfied in this case with $\left.v_{t}:=V\right)$. The goal of this paper is to try to explain to what extent the above assertions can be extended to the case when the vector field $V$ can be non smooth, and even strongly discontinuous, for instance just measurable. Namely, we are interested to establish whether one can say that a measurable vector field $V$ defines a reasonable flow of a given measure along the integral curves of $V$ and satisfying the continuity equation (1.2). Of course, the notion of an integral curve of $V$ must be now understood in a weaker sense, namely, as absolutely continuous Carathèodory solutions of (1.1) (i.e. the latter equations must be satisfied a.e. in $\mathbb{R}^{+}$). The following simple example shows however that we might not expect this to be possible for all measurable vector fields, because in fact some of them may admit no nonconstant integral curves.

Example 1.1. Let $K \subset[0,1]$ be a Cantor set of positive Lebesgue measure (or just any closed totally disconnected set of positive Lebesgue measure) and let $V(x):=\mathbf{1}_{K}(x)$. Then there is no absolutely continuous solution to the equation (1.1) different from constant curves $\theta(t) \equiv x \notin K$.

1991 Mathematics Subject Classification. 35R05, 35R06, 49Q15; 35F05, 35Q35, 35R15.

Key words and phrases. Flow of measures, continuity equation, metric current, measurable vector field, integral curve.

The first author is member of the GNAMPA group (Istituto Nazionale di Alta Matematica, Italy). The second author has been sponsored by the St.Petersburg State University grants \#6.60.1355.2016 and \#6.38.223.2014, by RFBR grant \#14-01-00534 and by the Government of Russian Federation grant \#074-U01. 
Looking at the above example from another point of view, one might guess that in fact to overcome the possible difficulty with the pointwise definition of the integral curve, it would be helpful to have the right "relaxation" of the notion of a smooth vector field. The idea of the appropriate weak concept comes from differential geometry. In fact, a classical smooth vector field can be viewed as a linear operator over the algebra of smooth functions over $E$ satisfying the Leibniz rule, or, in other words, the derivation (in this way one customarily identifies the vector field, i.e. a map $V: E \rightarrow E$ with the directional derivative along the latter). The correct weak analogue of this notion can be obtained by substituting smooth functions with appropriately less smooth ones. Namely, we will view the measurable vector field as a linear operator mapping Lipschitz functions into measurable ones (with respect to some measure), satisfying the Leibniz rule and some extra continuity assumption. This notion has been first introduced in [22] and adapted to metric measure spaces in [16] (for the detailed treatment of the subject as well as recent result related to it see also $[13,12])$. It is well-known that such vector fields can in fact be identified with one-dimensional metric currents introduced in [3]. It is the main goal of this paper to show that for a large class of such measurable vector fields, the notion of integral curves still makes sense, and the flow of a given measure along the respective integral curves can be defined in a reasonable way, and in particular, it satisfies the continuity equation (1.2) with some natural velocity field $v_{t}$ related to $V$.

Measurable vector fields and integral curves. The measurable vector fields (or, equivalently, one-dimensional metric currents) admitting the natural notion of "integral curves", called in this paper laminated, are those that can be represented as integrals of vector fields associated to single absolutely continuous curves in some possibly $\sigma$-finite measure $\eta$ over the space of curves $C\left(\mathbb{R}^{+} ; E\right)$. As shown in $[19,20]$, this class includes normal vector fields, i.e. the weak divergence of which is a signed Radon measure (in the Euclidean space this is a consequence of representation results for normal De Rham one-dimensional currents by Smirnov [21]), in which case there is a finite measure $\eta$ representing such a vector field. However, the class of laminated vector fields is strictly larger, although it does not include all measurable vector fields (in particular, the one from Example 1.1, see Remark A.12). Note that in view of Proposition A.4 a laminated vector field must have rather particular structure: namely (at least in a Euclidean space) it corresponds to a vector measure with the field of directions tangential to its mass measure in the sense of BouchittèButtazzo-Seppecher [9], which is not the case for generic measurable vector fields by Remark A.5. Our first main result is Theorem 5.1 which says in particular that in a Banach space $E$ with Radon-Nikodym property (e.g., in a finite-dimensional space)

- every laminated vector field $X$ has a pointwise representation as a pair of a "total variation" measure $m_{X}$ (or, equivalently, the mass measure of the respective metric current) and a Borel "field of directions", i.e. a Borel function $V: E \rightarrow E$ with $\|V(y)\|=1$ for $m_{X}$-a.e. $y \in E$ (\| $\cdot \|$ standing for the norm in $E$ ), such that

$$
(X \pi)(y)=\langle V(y), d \pi(y)\rangle, \quad \text { for } m_{X} \text {-a.e. } y \in E
$$

for every $\pi \in \operatorname{Lip}(E)$ quasi-differentiable function with bounded derivative $d \pi$ satisfying some rather weak continuity property (the respective class of functions being denoted by $\left.Q^{1}(E)\right),\langle\cdot, \cdot\rangle$ standing for the usual duality pairing between $E$ and its continuous dual; the map $V$ is unique up to $m_{X}$-a.e. equality;

- $\eta$-a.e. curve $\theta \in C\left(\mathbb{R}^{+} ; E\right)$ parameterized by arclength is an absolutely continuous integral curve of $X$ in the sense that when $E$ is strictly convex, then 
$\theta$ is a Carathéodory solution of (1.1) with $V$ the above "field of directions" of $X$ and $t \in(0, \ell(\theta))$, where $\ell(\theta)$ stands for the length of $\theta$; if $E$ is not strictly convex, then (1.1) has to be substituted by a differential inclusion.

Transportable measures and their flows. Let us describe in very rough and heuristic terms the notion of flow of a given measure $\mu$ by a given laminated vector field $X$ that we propose in this paper. The flow is seen as a movement of an ensemble of particles, each one moving along some integral curve of $X$. The movement of this ensemble is assumed to be given by a measure $\sigma$ over $C\left(\mathbb{R}^{+} ; E\right) \times \mathbb{R}^{+}$, so that, intuitively, $\sigma(\theta, s)$ stands for the number of particles which move along the curve $\theta \in C\left(\mathbb{R}^{+} ; E\right)$ at time $s \in \mathbb{R}^{+}$. Suppose that we set our timer at $t:=0$ and observe the distribution of the particles. Each particle at this instant is moving along some curve $\theta \in C\left(\mathbb{R}^{+} ; E\right)$ for some time $s \in \mathbb{R}^{+}$and hence is observed at the position $\beta_{0}(\theta, s):=\theta(s) \in E$, so that we observe the distribution of particles given by the measure $\mu:=\beta_{0 \#} \sigma$. After time $t \in \mathbb{R}^{+}$is elapsed (i.e. when our timer shows $t$ ), we observe the measure $\mu_{t}:=\beta_{t \#} \sigma$, where $\beta_{t}(\theta, s):=\theta(s+t) \in E$. The family $\left\{\mu_{t}\right\}_{t \in \mathbb{R}^{+}}$may be viewed thus as a flow of the measure $\mu=\mu_{0}$. In order that each particle in the flow of the measure $\mu$ be flowing along an integral curve of $X$, one has to request the projection of the measure $\sigma$ to the space of curves $C\left(\mathbb{R}^{+} ; E\right)$ to be absolutely continuous with respect to $\eta$. It is worth remarking that our notion of the measure $\sigma$ producing the flow is very similar to that of a randomized stopping time introduced in [18] and recently successfully employed in [6]. A curious feature of this definition of the flow is its sensibility to equivalent choices of distances in $E$ which is due to the same sensibility of the notion of "integral curves" to a measurable vector field; in fact, even for a finite-dimensional normed space different choices of the norm produce different representations of the same measurable vector field and hence different flows.

Clearly, there is a very strong non-uniqueness of the constructed flow due to both non-uniqueness of a measure $\eta$ representing the given vector field $X$ and also that of the measure $\sigma$ producing the flow. Part (but not all) of this non-uniqueness may be avoided by providing natural constructions of flows for particular subclasses of vector fields. We do this in Section 7 for so-called cyclic, or divergence free, and acyclic vector fields separately (it is worth keeping in mind that every vector field can be represented, though again not uniquely, as a sum of a cyclic one and an acyclic one).

More important, it is not guaranteed that for the given $\mu$ and $X$ (and $\eta$ representing $X$ ) the flow (i.e. the measure $\sigma$ producing the latter) in fact exists. When it exists, we say that $\mu$ is transportable by $X$. One might anyhow easily assume that each $\mu$ is transportable by every laminated vector field $X$ by admitting possibly very unnatural flows (in particular, such that every measure be not moving at all, that is, $\mu_{t}=\mu$ for all $t \in \mathbb{R}^{+}$). In order to avoid this we must not consider the measures $\eta$ over curves which charge constant curves (those concentrated over singletons). This produces physically reasonable flows but the transportability of all measures is sacrificed. In particular, for the measure $\mu$ to be transportable by $X$ it has to be concentrated over the support of $X$. We show that, given a laminated vector field $X$, still a lot of measures are transportable by $X$, in particular, the negative part of its weak divergence (if the latter is a finite measure), and its mass measure; and, moreover, every $\nu \ll \mu$ is transportable by $X$ if so is $\mu$ (see Proposition 4.2).

Continuity equations. The principal result of this paper regarding flows of transportable measures is Theorem 6.1 which shows in particular that in a Banach space with Radon-Nikodym property (e.g. in a finite-dimensional normed space), every flow $\mu_{t}$ corresponding to $X$ satisfies continuity equation (1.2) in the appropriately weak sense. Moreover, if the norm in $E$ is strictly convex, then the velocity field $v_{t}$ is collinear to $V$, the Borel map pointwise representing $X$ (in fact, $v_{t}=V\left(1-\varphi_{t}\right.$ ) 
where $\varphi_{t}: E \rightarrow[0,1]$ is some bounded function which may be nonzero due to the fact that some mass is stopped during the movement).

Note that the results established in this paper may be considered complementary to superposition principles obtained in [5, sections 7 and 8$]$ and in [2, theorem 12], although they look in the opposite direction: there one considers families of measures satisfying continuity equation (1.2) and shows that under appropriate conditions they have to "flow" along the trajectories of some ODE.

The basic notation as well as some useful auxiliary results on metric currents are reported in Appendix A. Some remarks on the representation of currents by curves are provided in Appendix A.3. Appendix B contains more or less folkloric technical results that are only provided for the readers' convenience as well as to explain some notation.

\section{NotATION AND PRELIMINARIES}

2.1. Framework and basic notation. The metric spaces we deal with in the sequel are always assumed to be complete. Unless explicitly stated otherwise, $E$ will stand for a complete metric space (our main results, Theorem 5.1 and Theorem 6.1, are among notable exceptions: there $E$ stands for a Banach space). For a $D \subset E$, we will denote by $\bar{D}$ its closure, by $\partial D$ its topological boundary, by $\mathbf{1}_{D}$ its characteristic function, by $\mathcal{H}^{k}$ the $k$-dimensional Hausdorff measure and let $D^{c}:=E \backslash D$. The notation $B_{r}(x) \subset E$ stands for the open ball of $E$ centered at $x \in E$ with radius $r>$ 0 . For real numbers $\alpha$ and $\beta$ we employ the usual brief notation $\alpha \vee \beta:=\max \{\alpha, \beta\}$ and $\alpha \wedge \beta:=\min \{\alpha, \beta\}$. By $\bar{e}_{i}, i=1, \ldots, n$ we denote the unit vectors along axis $x_{i}$ in $\mathbb{R}^{n}$. The notation $\mathcal{L}^{n}$ stands for the Lebesgue measure in $\mathbb{R}^{n}$.

If $E$ is a Banach space with norm $\|\cdot\|$, its dual will be denoted by $E^{\prime}$ and assumed to be equipped by the dual norm $\|\cdot\|^{\prime}$, the duality being denoted by $\langle\cdot, \cdot\rangle: E \times E^{\prime} \rightarrow \mathbb{R}$. The space $E$ will be also silently identified, if necessary, with its natural isometric embedding in $E^{\prime \prime}$. A Banach space $E$ is said to be strictly convex, if so is its unit ball.

2.2. Measures. The Borel $\sigma$-algebra of a metric space $E$ will be denoted by $\mathscr{B}(E)$. All the measures over a metric space $E$ we will consider in the sequel are Borel. We say that a positive measure $\mu$ over $E$ is concentrated over $D \in \mathscr{B}(E)$, if $\mu\left(D^{c}\right)=$ 0 . Further, all finite positive Borel measures we will be working with are silently assumed to be tight (i.e. concentrated over a $\sigma$-compact set), hence, in fact, Radon, since $E$ is assumed to be complete. This is true for all finite positive Borel measures if $E$ is Polish (i.e. complete separable), or, more generally, if the density character (i.e. the minimum cardinality of a dense subset) of $E$ is an Ulam number (see, e.g., proposition 7.2.10 from [8]). Note that it is consistent with the Zermelo-Fraenkel set theory to assume that the latter holds for every metric space. Anyhow, if $\mu$ is a tight positive finite Borel measure over $E$ then it is concentrated over a closed separable subspace $S \subset E$ (for $S$ one can take, for instance, the support supp $\mu$ of $\mu$, or the closed linear span of $\operatorname{supp} \mu$ if $E$ is a Banach space and one wants $S$ to be linear), and hence, in all such situations for the purposes of this paper, up to substituting $E$ by its separable subspace $S$ we may suppose, without loss of generality, that $E$ is separable itself. For two measures $\mu$ and $\nu$ over the same measurable space we use the standard notation $\nu \ll \mu$ to say that $\nu$ is absolutely continuous with respect to $\mu$ and $\nu \perp \mu$ to say that these measures are mutually singular. We say that $\mu$ and $\nu$ are equivalent, if $\mu \ll \nu \ll \mu$.

If a Borel measure $\mu$ over a metric space $E$ can be represented (we customarily use the term disintegrated) as

$$
\mu(B)=\int_{X} \mu_{x}(B) d \nu \quad \text { for all } B \in \mathscr{B}(E),
$$


where $\nu$ is a Borel measure over another metric space $X$ and each $\mu_{x}$ is a Borel measure over $E$, the map $x \in E \mapsto \mu_{x}(B)$ being $\nu$-measurable for all $B \in \mathscr{B}(E)$, we will write $\mu=\nu \otimes \mu_{x}$, in order to emphasize that the above disintegration formula may be seen as a generalization of the Fubini theorem for product measures.

2.3. Curves. Curves in a metric space $E$ will always be assumed parameterized over $\mathbb{R}^{+}$and continuous. The space of such curves $C\left(\mathbb{R}^{+} ; E\right)$ is equipped with uniform convergence over bounded intervals. The latter convergence is provided, for instance, by the distance

$$
d(u, v):=\sum_{k=1}^{+\infty} \frac{1}{2^{k}} \frac{d_{k}(u\llcorner[0, k], v\llcorner[0, k])}{1+d_{k}(u\llcorner[0, k], v\llcorner[0, k])},
$$

where $d_{k}$ stands for the usual uniform distance in $C([0, k] ; E)$ and $u\llcorner[a, b]$ stands for the restriction of the curve $u \in C\left(\mathbb{R}^{+} ; E\right)$ to the interval $[a, b]$. In the sequel we find it suitable to think of the space $C([a, b] ; E)$ of continuous functions over $[a, b]$ with values in $E$ (equipped with the usual uniform norm $\|\cdot\|_{\infty}$ ) as continuously embedded in $C\left(\mathbb{R}^{+} ; E\right)$ (equipped with the above distance) by the embedding map $\imath: C([a, b] ; E) \rightarrow C\left(\mathbb{R}^{+} ; E\right)$ defined by $\imath(\theta)(t):=\theta(a \wedge t \vee b)$. Note that once $E$ is separable, then $C\left(\mathbb{R}^{+} ; E\right)$ equipped with the latter distance is separable too (in fact, once $V_{k}$ is a dense subset of $C([0, k] ; E)$, then $\cup_{k} \imath\left(V_{k}\right)$ is dense in $\left.C(\mathbb{R}+; E)\right)$.

For an absolutely continuous curve $\theta \in C\left(\mathbb{R}^{+} ; E\right)$ we let

$$
\ell(\theta)=\int_{\mathbb{R}^{+}}|\dot{\theta}|(t) d t
$$

where $|\dot{\theta}|(t)$ stands for the metric derivative of $\theta$ at $t \in \mathbb{R}^{+}$, which exists a.e. in $\mathbb{R}^{+}$ and is everywhere in the sequel silently assumed to be locally integrable as part of definition of absolutely continuous curves. Note that if $E$ is a Banach space with Radon-Nikodym property, then in fact the derivative $\dot{\theta}(t)$ is well-defined for a.e. $t \in \mathbb{R}^{+}$(with the limit in the definition of the derivative intended in the sense of the norm), and hence $\|\dot{\theta}(t)\|=|\dot{\theta}|(t)$ for a.e. $t \in \mathbb{R}^{+}$. The value $\ell(\theta)$ is usually referred to as parametric length of $\theta$. An absolutely continuous curve $\theta \in C\left(\mathbb{R}^{+} ; E\right)$ will be called rectifiable, if $\ell(\theta)<+\infty$. For every rectifiable curve $\theta$ the sequence $\left\{\theta\left(t_{k}\right)\right\}$ for $t_{k} \rightarrow+\infty$ is Cauchy, since for $t_{k} \leq t_{j}$ one has

$$
d\left(\theta\left(t_{k}\right), \theta\left(t_{j}\right)\right) \leq \ell\left(\theta\left\llcorner\left[t_{k}, t_{j}\right]\right)=\ell\left(\theta\left\llcorner\left[0, t_{k}\right]\right)-\ell\left(\theta\left\llcorner\left[0, t_{j}\right]\right) \rightarrow 0\right.\right.\right.
$$

as $k, j \rightarrow+\infty$. Thus the limit

$$
\operatorname{end}(\theta):=\lim _{t \rightarrow+\infty} \theta(t)
$$

is well defined (we will refer to it as ending point of $\theta$ ). The starting point $\operatorname{start}(\theta)$ is just $\operatorname{start}(\theta):=\theta(0)$. The function $e_{t}: C\left(\mathbb{R}^{+} ; E\right) \rightarrow E$ is defined by $e_{t}(\theta):=\theta(t)$, $t \in \mathbb{R}^{+}$. We will say that a rectifiable curve $\theta$ is parameterized by arclength, if $|\dot{\theta}|(t)=1$ for a.e. $t \in[0, \ell(\theta)]$ and $|\dot{\theta}|(t)=0$ for a.e. $t \geq \ell(\theta)$ (so that, in particular, $\operatorname{start}(\theta)=\theta(0)$ and end $(\theta)=\theta(t)$ for all $t \geq \ell(\theta))$.

2.4. Spaces. For a metric space $E$ we denote by $\operatorname{Lip}(E)\left(\operatorname{resp} \operatorname{Lip}_{b}(E)\right)$ the set of all real-valued Lipschitz maps (resp. all bounded Lipschitz maps), and by $C_{b}(E)$ the set of all real-valued bounded continuous maps equipped with the uniform norm. The notation $L^{p}(E ; \mu)$ (resp. $L_{l o c}^{p}(E ; \mu)$ ) will stand for the usual Lebesgue space of (classes of $\mu$-a.e. equal) real valued maps over $E$ integrable (resp. locally integrable) with power $p \in[1 ;+\infty)$ with respect to the positive (possibly $\sigma$-finite) Borel measure $\mu$ (or $\mu$-essentially bounded for $p=+\infty$ ). If $(\Omega, \Sigma, \mu)$ is a measure space with some positive $\sigma$-finite measure $\mu$, and $E$ is a Banach space, then $L^{1}(\Omega, \mu ; E)$ stands for the space of Bochner integrable functions $f: \Omega \rightarrow E$. By $C^{1}\left(\mathbb{R}^{n}\right)$ (resp. $C_{0}^{1}\left(\mathbb{R}^{n}\right)$ ) we denote as usual the set of continuously differentiable functions (resp. continuously differentiable with compact support) in $\mathbb{R}^{n}$. 
For a Banach space $E$, the function $\varphi: E \rightarrow \mathbb{R}$ is called quasi-differentiable, if for every $x \in E$ there is a functional $d \varphi(x) \in E^{\prime}$ such that

$$
\varphi(\theta(t+\varepsilon))=\varphi(\theta(t))+\langle\dot{\theta}(t), d \varphi(\theta(t))\rangle \varepsilon+o(\varepsilon)
$$

as $\varepsilon \rightarrow 0^{+}$whenever $\theta \in C\left(\mathbb{R}^{+} ; E\right)$ is differentiable at $t \in \mathbb{R}^{+} \backslash\{0\}$ and $\theta(t)=x$. This notion of differentiability is slightly stronger then Gâteaux one but strictly weaker than Fréchet one. By $Q^{1}(E)$ we denote then the set of quasi-differentiable functions $\varphi: E \rightarrow \mathbb{R}$ such that $\|d \phi(\cdot)\|^{\prime}$ is uniformly bounded and the map $(x, y) \mapsto\langle y, d \varphi(x)\rangle$ is continuous. Note that $Q^{1}(E) \subset \operatorname{Lip}(E)$, since for $u \in Q^{1}(E)$ one has

$$
\begin{aligned}
|u(y)-u(x)| & =\left|\int_{0}^{1} \frac{d}{d t} u((1-t) x+t y) d t\right|=\left|\int_{0}^{1}\langle y-x, d u((1-t) x+t y)\rangle d t\right| \\
& \leq \int_{0}^{1}\|y-x\| \cdot\|d u((1-t) x+t y)\|^{\prime} d t \leq C\|y-x\|,
\end{aligned}
$$

where $C>0$ is such that $\|d u(z)\|^{\prime} \leq C$ for all $z \in E$.

2.5. Metric currents. For metric currents we use the notation from [19] which is taken from [3], except the notation for the mass measure. In particular, $D^{k}(E)=$ $\operatorname{Lip}_{b}(E) \times(\operatorname{Lip}(E))^{k}$ stands for the space of metric $k$-forms, its elements (i.e. $k$-forms) being denoted by $f d \pi$, where $f \in \operatorname{Lip}_{b}(E), \pi \in(\operatorname{Lip}(E))^{k}, \mathcal{M}_{k}(E)$ stands for the space of $k$-dimensional metric currents, $\mathbb{M}(T)$ stands for the mass of a current $T, \partial T$ for its boundary and $m_{T}$ stands for the mass measure associated to this current (the latter is denoted by $\|T\|$ in [3] and $m_{T}$ in [19, 20], but here we introduce a different notation in order to avoid confusion between mass measures of currents, norms, and flows of measures). As we assume generally for every finite Borel measures, we presume by default that the mass measures of the currents are all tight, i.e. concentrated over $\sigma$-compact sets (which is automatically true if $E$ is separable, or, more generally, when the density character of $E$ is Ulam number). In fact, all the statements on metric currents from [3] remain valid under this assumption.

We also use the notion of subcurrents from [19]. Namely an $S \in \mathcal{M}_{k}(E)$ is called subcurrent of a $T \in \mathcal{M}_{k}(E)$, written $S \leq T$, if $\mathbb{M}(S)+\mathbb{M}(T-S)=\mathbb{M}(T)$. Finally, the current $T \in \mathcal{M}_{k}(E)$ is normal, if $\partial T \in \mathcal{M}_{k-1}(E)$, i.e. is still a metric current.

The one-dimensional current associated to a Lipschitz curve $\theta:[a, b] \rightarrow E$ is denoted by $\llbracket \theta \rrbracket$, namely,

$$
\llbracket \theta \rrbracket(f d \pi):=\int_{a}^{b} f(\theta(t)) d \pi(\theta(t))
$$

for every $f d \pi \in D^{1}(E)$. Recall that $\mathbb{M}(\llbracket \theta \rrbracket) \leq \ell(\theta)$. The sequence of currents $\left\{T_{\nu}\right\} \subset \mathcal{M}_{k}(E)$ is said to converge weakly to a $T \in \mathcal{M}_{k}(E)$, written $T_{\nu} \rightarrow T$, if $T_{\nu}(\omega) \rightarrow T(\omega)$ as $\nu \rightarrow \infty$ for all $\omega \in D^{k}(E)$.

\section{VECTOR FIELDS AS DERIVATIONS}

In the sequel we will be working with the following notion of a measurable vector field introduced in [22]. We use it in the form adapted to metric measure spaces in [16].

Definition 3.1. Let $\mu$ be a Borel measure over $E$. We will call a bounded linear operator $X: \operatorname{Lip}_{b}(E) \rightarrow L^{\infty}(E ; \mu)$ a $\mu$-vector field (or measurable vector field or even just vector field when we do not need to mention the underlying measure), if it satisfies

- (Leibniz rule) $X(f g)=f X g+g X f$ for all $\{f, g\} \subset \operatorname{Lip}_{b}(X)$

- (weak continuity) $\lim _{k} X f_{k}=X f$ in the weak* sense of $L^{\infty}(E ; \mu)$, i.e., whenever $\lim _{k} f_{k}(x)=f(x)$ for all $x \in E$ and $\sup _{k}\left(\left\|f_{k}\right\|_{\infty}+\operatorname{Lip} f_{k}\right)<+\infty$. 
It is worth mentioning that operators over function algebrae satisfying Leibniz rule are usually called derivations.

As mentioned in Appendix A.2, a $\mu$-vector field $X$ can be extended by locality to the whole $\operatorname{Lip}(E)$ (and we do not change the name for this extension). Further, by Proposition A.8 there is a one-to-one correspondence between measurable vector fields and metric currents, namely, every $\mu$-vector field $X$ over $E$ defines a metric current $T \in \mathcal{M}_{1}(E)$ by the formula

$$
T(f d \pi):=\int_{E} f X \pi d \mu
$$

with $m_{T} \leq C \mu$ and, vice-versa, every $T \in \mathcal{M}_{1}(E)$ with $m_{T} \leq C \mu$ defines a unique $\mu$-vector field $X$ over $E$ such that (3.1) holds. This gives a possibility to identify onedimensional metric currents with measurable vector fields, which we will always do in the sequel. In particular, we may attribute the terminology of currents to vector fields, e.g. calling the vector field normal, if so is the respective current.

We introduce also the following notion which will be important in what follows.

Definition 3.2. We will say that a current $T \in \mathcal{M}_{1}(E)$ is represented by a $\sigma$-finite Borel measure $\eta$ over $C\left(\mathbb{R}^{+} ; E\right)$ without cancelation of mass, if

$$
\begin{aligned}
T(\omega) & =\int_{C\left(\mathbb{R}^{+} ; E\right)} \llbracket \theta \rrbracket(\omega) d \eta(\theta) \text { for all } \omega \in D^{1}(E), \\
\mathbb{M}(T) & =\int_{C\left(\mathbb{R}^{+} ; E\right)} \ell(\theta) d \eta(\theta) .
\end{aligned}
$$

$A \mu$-vector field $X$ over $E$ will be said to be represented by a $\sigma$-finite Borel measure $\eta$ over $C\left(\mathbb{R}^{+} ; E\right)$, if so is the respective metric current $T \in \mathcal{M}_{1}(E)$ defined by (3.1). Such vector fields and currents will be further called laminated.

\section{Transportable measures AND THEIR FLOWS}

Define $\beta_{t}: C\left(\mathbb{R}^{+} ; E\right) \times \mathbb{R}^{+} \rightarrow E$ by the formula

$$
\beta_{t}(\theta, s):=\theta(t+s)
$$

so that in particular $\beta_{0}(\theta, s)=\theta(s)$. Consider also the projection

$$
q:(\theta, s) \in C\left(\mathbb{R}^{+} ; E\right) \times \mathbb{R}^{+} \mapsto \theta \in C\left(\mathbb{R}^{+} ; E\right) .
$$

Following the idea presented in the Introduction, we give the following definition.

Definition 4.1. A finite positive Borel measure $\mu$ over $E$ will be called transportable by a $\sigma$-finite Borel measure $\eta$ over $C\left(\mathbb{R}^{+} ; E\right)$, through a finite Borel measure $\sigma$ over $C\left(\mathbb{R}^{+} ; E\right) \times \mathbb{R}^{+}$, if

$$
\begin{array}{r}
\beta_{0 \#} \sigma=\mu, \\
q_{\#} \sigma \ll \eta .
\end{array}
$$

We will also say that $\mu$ is transportable by $\eta$, if there is a $\sigma$ satisfying (4.1). The family of measures $\left\{\mu_{t}\right\}_{t \in \mathbb{R}^{+}}$defined by

$$
\mu_{t}:=\beta_{t \#} \sigma,
$$

will then be called the flow of measures corresponding to $\eta$.

Further, the measure $\mu$ will be called transportable by a laminated measurable vector field $X$ (or, equivalently, by the respective current $T \in \mathcal{M}_{1}(E)$ ), if there is a $\sigma$ finite Borel measure $\eta$ over $C\left(\mathbb{R}^{+} ; E\right.$ ) representing $X$ (resp. $T$ ) without cancelation of mass, which does not charge constant curves, such that $\mu$ is transportable by $\eta$. 
Assume that a Borel measure $\mu$ over $E$ be transportable by a Borel measure $\eta$ over $C\left(\mathbb{R}^{+} ; E\right)$ through a Borel measure $\sigma$ over $C\left(\mathbb{R}^{+} ; E\right) \times \mathbb{R}^{+}$(i.e. the latter satisfies (4.1)). Clearly, with this definition one has $\mu_{t}(E)=\mu(E), \mu_{0}=\mu$ and the family $\left\{\mu_{t}\right\}$ forms a narrowly continuous curve in the space of finite Borel measures over $E$. Further, by disintegration theorem we have

$$
\sigma=\mu \otimes \eta_{x}
$$

where $\left\{\eta_{x}\right\}_{x \in E}$ is a family of Borel probability measures over $C\left(\mathbb{R}^{+} ; E\right) \times \mathbb{R}^{+}$, concentrated over

$$
\beta_{0}^{-1}(x):=\left\{(\theta, s) \in C\left(\mathbb{R}^{+} ; E\right) \times \mathbb{R}^{+}: \theta(s)=x\right\} .
$$

Setting $h_{x, t}:=\beta_{t \#} \eta_{x}$, one has in particular

$$
\mu_{t}=\beta_{t \#} \sigma=\mu \otimes h_{x, t},
$$

and for every $t \in \mathbb{R}^{+}$the map $x \in E \mapsto h_{x, t}$ is weakly measurable. In other words,

$$
\mu_{t}(B):=\int_{E} h_{x, t}(B) d \mu(x)
$$

for every Borel $B \subset E$.

We study now the notion of transportability of the measures introduced above. The following easy statement is valid.

Proposition 4.2. Assume that a Borel measure $\mu$ over $E$ be transportable by a Borel measure $\eta$ over $C\left(\mathbb{R}^{+} ; E\right)$. Then every finite measure $\psi \ll \mu$ is transportable by $\eta$.

Proof. By the Radon-Nikodym theorem we may write $\psi=\alpha \mu$ with a measurable $\alpha: E \rightarrow \mathbb{R}^{+}$. Let $\sigma$ be a Borel measure over $C\left(\mathbb{R}^{+} ; E\right) \times \mathbb{R}^{+}$satisfying (4.1). We define the Borel measure $\tau$ over $C\left(\mathbb{R}^{+} ; E\right) \times \mathbb{R}^{+}$by setting $\tau:=\left(\alpha \circ \beta_{0}\right) \sigma$. Note that $\tau$ is a $\sigma$-finite Borel measure (in fact, finite, if so is $\psi$ ), because

$$
\int_{\beta_{0}^{-1}(B)} \alpha \circ \beta_{0} d \sigma=\int_{B} \alpha d \beta_{0 \#} \sigma=\int_{B} \alpha d \mu=\psi(B)
$$

for every Borel $B \subset E$. Therefore, $\tau \ll \sigma$, hence $q_{\#} \tau \ll q_{\#} \sigma \ll \eta$. On the other hand, by construction one has $\beta_{0 \#} \tau=\psi$ by (4.2). Therefore, $\psi$ is transportable by $\eta$ through $\tau$.

Note that in Definition 4.1 in the notion of transportability by a laminated measurable vector field $X$ it has been requested that a measure $\eta$ over curves representing $X$ without cancelation of mass does not charge constant curves (i.e. curves, the images of which are singletons). If this requirement were dropped, then every measure $\mu$ would be transported by every laminated measurable vector field $X$. In fact, let $\eta$ be an arbitrary $\sigma$-finite Borel measure over $C\left(\mathbb{R}^{+} ; E\right)$ representing $X$ without cancelation of mass, and let $f: E \rightarrow C\left(\mathbb{R}^{+} ; E\right)$ be defined by $f(x)(t):=x$ (i.e. $f$ maps a point $x \in E$ to the constant curve $\theta(t)=x$ ). Letting $\eta^{\prime}:=f_{\#} \mu$ we have that $\eta+\eta^{\prime}$ still represents the same measurable vector field without cancelation of mass, and $\mu$ is transportable by $\eta+\eta^{\prime}$, say, by a measure $\sigma:=\eta^{\prime} \otimes \delta_{0}$ over $C\left(\mathbb{R}^{+} ; E\right) \times \mathbb{R}^{+}$. However, the respective flow is in fact absolutely unreasonable since one has $\mu_{t}=\mu$ for all $t \in \mathbb{R}^{+}$, which has nothing to do with the vector field in consideration. Thus we have to sacrifice the possibility to transport all the measures in order to have reasonable flows. In fact, not all the measures are transportable by a given measurable vector field as the following statement shows.

Proposition 4.3. If $\mu$ is a measure over $E$ transportable by a laminated measurable vector field $X$ (or, equivalently, by the respective current $T \in \mathcal{M}_{1}(E)$ ), then $\mu$ is concentrated over $\operatorname{supp} T$ (i.e. $\operatorname{supp} \mu \subset \operatorname{supp} T)$. 
Proof. Let $\eta$ be a $\sigma$-finite Borel measure over $C\left(\mathbb{R}^{+} ; E\right) \times \mathbb{R}^{+}$representing $T$ without cancelation of mass, and not charging constant curves, such that $\mu$ is transportable by $\eta$ through some measure $\sigma$ over $C\left(\mathbb{R}^{+} ; E\right) \times \mathbb{R}^{+}$. By Remark A.9, one has then $\theta \subset \operatorname{supp} T$ in the sense of traces for $\eta$-a.e. $\theta$, i.e. $\theta(t) \in \operatorname{supp} T$ for $\eta$-a.e. $\theta \in C\left(\mathbb{R}^{+} ; E\right)$ and all $t \in \mathbb{R}^{+}$, hence for $\sigma$-a.e. $(\theta, t) \in C\left(\mathbb{R}^{+} ; E\right) \times \mathbb{R}^{+}$. Thus $\beta_{0 \#} \sigma=\mu$ is concentrated over $\operatorname{supp} T$.

We now give some examples of transportable measures.

Example 4.4. Assume $\eta$ be a finite measure. Then $\mu:=\operatorname{start}_{\#} \eta=e_{0 \#} \eta$ is transportable by $\eta$ through $\sigma:=\eta \otimes \delta_{0}$. In fact,

$$
\begin{aligned}
\int_{E} u(x) d\left(\beta_{0 \#} \sigma\right)(x) & =\int_{E} u(x) d \beta_{0 \#}\left(\eta \otimes \delta_{0}\right)(x) \\
& =\int_{C\left(\mathbb{R}^{+} ; E\right) \times \mathbb{R}^{+}} u\left(\beta_{0}(\theta, s)\right) d\left(\eta \otimes \delta_{0}\right)(\theta, s) \\
& =\int_{C\left(\mathbb{R}^{+} ; E\right) \times \mathbb{R}^{+}} u(\theta(s)) d\left(\eta \otimes \delta_{0}\right)(\theta, s)=\int_{C\left(\mathbb{R}^{+} ; E\right)} u(\theta(0)) d \eta(\theta) \\
& =\int_{E} u(x) d\left(e_{0 \#} \eta\right)(x)=\int_{E} u(x) d \mu(x)
\end{aligned}
$$

for every Borel function $u$ over $E$, so that $\beta_{0 \#} \sigma=\mu$, and hence $\sigma$ satisfies (4.1).

We have now that each $\eta_{x}$ is concentrated over

$$
e_{0}^{-1}(x) \times\{0\}=\left\{\theta \in C\left(\mathbb{R}^{+} ; E\right): \theta(0)=x\right\} \times\{0\} \subset C\left(\mathbb{R}^{+} ; E\right) \times \mathbb{R}^{+},
$$

and

$$
\begin{aligned}
h_{x, t}(B) & =\eta_{x}\left(\left\{(\theta, s) \in C\left(\mathbb{R}^{+} ; E\right) \times \mathbb{R}^{+}: \theta(s+t) \in B\right\}\right) \\
& =\eta_{x}\left(\left\{\theta \in C\left(\mathbb{R}^{+} ; E\right): \theta(t) \in B\right\} \times\{0\}\right)=\eta_{x}\left(e_{t}^{-1}(B) \times\{0\}\right) .
\end{aligned}
$$

Finally, one has $\mu_{t}=e_{t \#} \eta$. In fact,

$$
\begin{aligned}
\mu_{t}(B) & =\int_{E} h_{x, t}(B) d \mu(x)=\int_{E} \eta_{x}\left(e_{t}^{-1}(B) \times\{0\}\right) d \mu(x) \\
& =\left(\eta \otimes \delta_{0}\right)\left(e_{t}^{-1}(B) \times\{0\}\right)=\eta\left(e_{t}^{-1}(B)\right)=\left(e_{t \#} \eta\right)(B)
\end{aligned}
$$

for all Borel $B \subset E$.

Another important example is the following one.

Example 4.5. Let $\eta$ be a $\sigma$-finite measure over $C\left(\mathbb{R}^{+} ; E\right)$ such that $\eta$-a.e. $\theta \in$ $C\left(\mathbb{R}^{+} ; E\right)$ is parameterized by arclength, and

$$
\int_{C\left(\mathbb{R}^{+} ; E\right)} \ell(\theta) d \eta(\theta)<+\infty .
$$

Then the finite measure $\mu$ over $E$ defined by

$$
\begin{aligned}
\mu & :=\eta \otimes \theta_{\#}\left(\mathcal{L}^{1}\llcorner[0, \ell(\theta)]),\right. \text { i.e. } \\
\mu(B) & =\int_{C\left(\mathbb{R}^{+} ; E\right)} \mathcal{L}^{1}\left(\theta^{-1}(B) \cap[0, \ell(\theta)]\right) d \eta(\theta),
\end{aligned}
$$

for every Borel set $B \subset E$, is transportable by $\eta$. In fact, setting $\sigma:=\eta \otimes \mathcal{L}^{1}\llcorner[0, \ell(\theta)]$, one has

$$
\begin{aligned}
\left(\beta_{0 \#} \sigma\right)(B) & =\sigma\left(\left\{(\theta, s) \in C\left(\mathbb{R}^{+} ; E\right) \times \mathbb{R}^{+}: \theta(s) \in B\right\}\right) \\
& =\int_{C\left(\mathbb{R}^{+} ; E\right)} \mathcal{L}^{1}\left(\theta^{-1}(B) \cap[0, \ell(\theta)]\right) d \eta(\theta)=\mu(B)
\end{aligned}
$$

for every Borel $B \subset E$. 
In particular, if $\eta$ represents some laminated current $T \in \mathcal{M}_{1}(E)$ without cancelation of mass, while $\eta$-a.e. $\theta \in C\left(\mathbb{R}^{+} ; E\right)$ is parameterized by arclength, then the mass measure $m_{T}$ is transportable by $\eta$ through $\sigma:=\eta \otimes \mathcal{L}^{1}\llcorner[0, \ell(\theta)]$, because clearly in this case one has $\mu=m_{T}$.

It is worth mentioning that the measure $\mu$ defined by (4.3) can be interpreted as the transport density. This notion is frequently used in the context of the MongeKantorovich optimal transportation problem (i.e. when $\eta$ and/or $T$ represent the solution to the latter problem) $[1,10]$.

Note that the above Example 4.5 provides a measure transportable by a measurable vector field by any measure $\eta$ representing the latter without cancelation of mass. In general this is not the case, i.e. if a measure is transportable by a measurable vector field, it is not true that it is transportable by all measures $\eta$ representing the latter without cancelation of mass as the following example shows.

Example 4.6. If $E:=\mathbb{R}, T:=\llbracket[0,1] \rrbracket$, then the measure $\mu:=\delta_{0}$ is transportable by $\eta:=\delta_{\theta}$ with $\theta:=[0,1]$ (i.e. $\theta(t):=t$ for all $t \in[0,1]$ ) by Example 4.4. However, $\mu$ is not transportable by $\eta^{\prime}:=\sum_{k=1}^{\infty} \delta_{\theta_{k}}$ (note that this measure is not finite) with $\theta_{k}:=\left[1 / 2^{k+1}, 1 / 2^{k}\right]\left(\right.$ i.e. $\theta_{k}(t):=(1-t) / 2^{k+1}+t / 2^{k}$ for all $\left.t \in[0,1]\right)$, while both $\eta$ and $\eta^{\prime}$ represent $T$ without cancelation of mass.

We will now give an example of a measure not transportable by a given measure $\eta$ over $C\left(\mathbb{R}^{+} ; E\right)$.

Example 4.7. Let $E:=\mathbb{R}^{2}$ and $\eta:=\mathcal{L}^{1}\left\llcorner[0,2] \otimes \delta_{\theta_{y}}\right.$, where $\theta_{y}(t):=(t \wedge 1, y)$ for each $y \in[0,2]$ (with the parameter $t \in \mathbb{R}^{+}$). This measure represents the current $\left(\mathcal{L}^{2}\llcorner[0,1] \times[0,2]) \wedge \bar{e}_{1}\right.$ without cancelation of mass. Let $A:=(0,1)=\theta_{1}(0)$ (see figure 4$)$. Then $\mu:=\delta_{A}$ is not transportable by $\eta$. In fact, suppose the contrary, i.e. the existence of a measure $\sigma$ over $C\left(\mathbb{R}^{+} ; E\right) \times \mathbb{R}^{+}$satisfying (4.1). Representing $\sigma:=\tilde{\eta} \otimes \lambda_{\theta}$, where $\tilde{\eta}:=q_{\#} \sigma$, we get that $\tilde{\eta}$ is concentrated over $\left\{\theta_{y}: y \in[0,2]\right\}$ (because $\tilde{\eta} \ll \eta$ ) and $\lambda_{\theta}=0$ for all $\theta \neq \theta_{1}$ because $\mu=\beta_{0 \#} \sigma$ is concentrated over a singleton $A$ which is the starting point of $\theta_{1}$. This means $\sigma=0$ giving the obvious contradiction $\mu=0$.

On the contrary, letting again $E:=\mathbb{R}^{2}$ and

$$
\eta^{\prime}:=\mathcal{L}^{1}\left\llcorner[-1,1] \otimes \delta_{\theta_{k}^{\prime}},\right.
$$

where $\theta_{k}^{\prime}(t):=(t \wedge 1, k(t \wedge 1))$ with the parameter $t \in \mathbb{R}^{+}$, and $k \in[-1,1]$, we have that $\eta^{\prime}$ represents some normal current $T^{\prime}$ (the one solving the optimal mass transportation problem of minimizing $\mathbb{M}(T)$ among normal currents $T$ satisfying $\partial T=\mathcal{H}^{1}\left\llcorner I-2 \delta_{O}\right.$, where $I$ stands for the segment with endpoints $(1,-1)$ and $(1,1))$. The latter current is concentrated over the triangle with vertices $O,(1,-1)$ and $(1,1)$ and has the field of directions $v$ given by $v(x, y)=(x, y / x) /|(x, y / x)|$ (see figure 4$)$. Then $\delta_{O}$ is transportable by $\eta^{\prime}$ by Example 4.4.

Remark 4.8. It is worth remarking that if the finite Borel measure $\mu$ is transportable by a measurable vector field corresponding to a laminated current $T$, then there is a normal current $S \leq T, S \neq 0$, and a finite Borel measure $\nu$ over $E$ such that $\partial S=\nu-\tilde{\mu}$, where $\tilde{\mu}$ is equivalent to $\mu$. In fact, in this case there is a $\sigma$-finite Borel measure $\eta$ over $C\left(\mathbb{R}^{+} ; E\right)$ representing $T$ without cancelation of mass and a Borel measure $\sigma$ over $C\left(\mathbb{R}^{+} ; E\right) \times \mathbb{R}^{+}$satisfying (4.1). Disintegrating $\sigma=\alpha \eta \otimes \lambda_{\theta}$, where $\alpha \in L^{1}\left(C\left(\mathbb{R}^{+} ; E\right), \eta\right)$ and each $\lambda_{\theta}$ is a Borel probability measure over $\mathbb{R}^{+}$, define

$$
\begin{aligned}
\tilde{\eta} & :=\frac{\alpha}{|\alpha|} \eta=\mathbf{1}_{\{\alpha>0\}} \eta, \quad \tilde{\sigma}:=\tilde{\eta} \otimes \lambda_{\theta}, \\
S & :=\int_{C\left(\mathbb{R}^{+} ; E\right) \times \mathbb{R}^{+}} \llbracket \theta\llcorner[s,+\infty) \rrbracket d \tilde{\sigma}(\theta, s),
\end{aligned}
$$



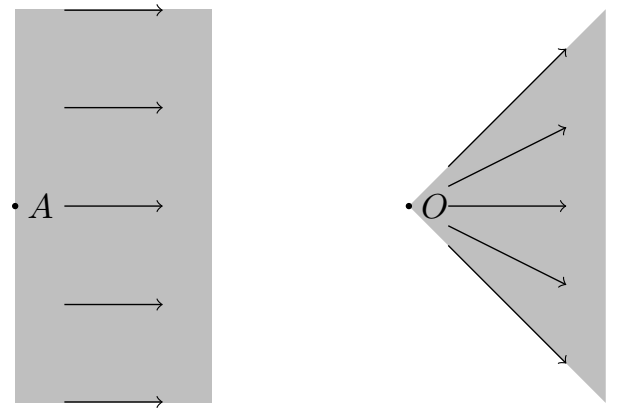

Figure 1. The measure $\delta_{A}$ is not transportable, while $\delta_{O}$ is transportable.

and keeping in mind that $\tilde{\eta}$ represents the laminated current $T_{\tilde{\eta}}$ without cancelation of mass by Lemma A.15, we get from Lemma A.17 (applied with $T_{\tilde{\eta}}$ and $\tilde{\sigma}$ instead of $T$ and $\sigma$ respectively) that $S \leq T_{\tilde{\eta}} \leq T$ (the latter inequality being due to the fact that $\tilde{\eta} \leq \eta)$. At last, one has

$$
\begin{aligned}
\partial S & =\int_{C\left(\mathbb{R}^{+} ; E\right) \times \mathbb{R}^{+}}\left(\delta_{\operatorname{end}(\theta)}-\delta_{\theta(s)}\right) d \tilde{\sigma}(\theta, s) \\
& =\int_{C\left(\mathbb{R}^{+} ; E\right)} \delta_{\operatorname{end}(\theta)} d \tilde{\eta}(\theta)-\int_{C\left(\mathbb{R}^{+} ; E\right) \times \mathbb{R}^{+}} \delta_{\theta(s)} d \tilde{\sigma}(\theta, s),
\end{aligned}
$$

but

$$
\begin{aligned}
\int_{E} f(x) d(\partial S)(x) & =\int_{C\left(\mathbb{R}^{+} ; E\right)} f(\operatorname{end}(\theta)) d \tilde{\eta}(\theta)-\int_{C\left(\mathbb{R}^{+} ; E\right) \times \mathbb{R}^{+}} f(\theta(s)) d \tilde{\sigma}(\theta, s) \\
& =\int_{E} f(x) d\left(\operatorname{end}_{\#} \tilde{\eta}-\beta_{0 \#} \tilde{\sigma}\right)(x)
\end{aligned}
$$

for every $f \in C_{b}(E)$, so that $\partial S=\operatorname{end}_{\#} \tilde{\eta}-\beta_{0 \#} \tilde{\sigma}$, and it remains to observe that for $\tilde{\mu}:=\beta_{0 \#} \tilde{\sigma}$ one has $\tilde{\mu} \ll \mu \ll \tilde{\mu}$ as claimed.

Let us illustrate the above Remark 4.8 by the following easy example.

Example 4.9. Let $E:=\mathbb{R}, T=\llbracket[0,1] \rrbracket, \eta:=\delta_{\theta}$, where $\theta:=[0,1]$, and $\mu=$ $m_{T}=\mathcal{L}^{1}\left\llcorner[0,1]\right.$. Set $S:=\int_{0}^{1} \llbracket[s, 1] \rrbracket d s$. Clearly, $T-S=\int_{0}^{1} \llbracket[0, s] \rrbracket d s$, so that $\mathbb{M}(S) \leq \int_{0}^{1}(1-s) d s=1 / 2$ and analogously $\mathbb{M}(T-S) \leq 1 / 2$, which gives $\mathbb{M}(S)+$ $\mathbb{M}(T-S) \leq 1=\mathbb{M}(T)$, and hence $S \leq T$. Further,

$$
\partial S=\int_{0}^{1}\left(\delta_{1}-\delta_{s}\right) d s=\delta_{1}-\int_{0}^{1} \delta_{s} d s=\delta_{1}-\mu,
$$

because

for every $f \in C_{b}(E)$.

$$
\int_{E} f(x) d(\partial S)^{-}(x)=\int_{0}^{1} f(s) d s=\int_{E} f d \mu
$$

As a partial converse to the above Remark 4.8 we may claim the following easy statement.

Proposition 4.10. For a normal current $T \in \mathcal{M}_{1}(E)$, if there is a normal current $S \leq T$ such that $\mu \ll(\partial S)^{-}$, then the measure $\mu$ is transportable by $T$.

Proof. Let $T$ and $S \leq T$ be normal one-dimensional currents. It is enough to show that $(\partial S)^{-}$is transportable by $T$ and then refer to Proposition 4.2 . Let $\eta$ be a finite Borel measure over $C\left(\mathbb{R}^{+} ; E\right)$ representing $S$ without cancelation of mass and concentrated on curves parameterized, say (for the sake of definiteness), over $[0,1]$ (i.e. being constant outside of this interval). By Example 4.4, one has that $e_{0 \#} \eta$ 
is transportable by $\eta$. But since $\partial S=e_{1 \#} \eta-e_{0 \#} \eta$, we have $(\partial S)^{-} \leq e_{0 \#} \eta$, and thus $(\partial S)^{-}$is transportable by $\eta$ again by Proposition 4.2 . Let now $\eta^{\prime}$ be a finite Borel measure over $C\left(\mathbb{R}^{+} ; E\right)$ representing $R:=T-S$ without cancelation of mass. Then, clearly, $\eta+\eta^{\prime}$ represents $T$ without cancelation of mass, and since $(\partial S)^{-}$is transportable by $\eta$, it is also transportable by any bigger measure, in particular, by $\eta+\eta^{\prime}$, hence is transportable by $T$, which concludes the proof.

In a particular case when the metric space $E$ is isometrically embedded in a strictly convex Banach space with Radon-Nikodym property, we are able to give the following more precise characterization of transportability condition and the flow it generates.

Proposition 4.11. Let $T \in \mathcal{M}_{1}(E)$ be a laminated current, where $E$ is isometrically embedded into a strictly convex Banach space with Radon-Nikodym property, $\mu$ be a finite Borel measure over $E$ transportable by $T$ through a finite Borel measure $\sigma$ over $C\left(\mathbb{R}^{+} ; E\right) \times \mathbb{R}^{+}$such that

$$
\int_{C\left(\mathbb{R}^{+} ; E\right)} \ell(\theta) d\left(q_{\#} \sigma\right)(\theta)<+\infty
$$

the respective flow of $\mu$ being given by the family of measures $\left\{\mu_{t}\right\}$ with $\mu_{0}=\mu$. Then there is a $\gamma \in L^{1}\left(E ; m_{T}\right)$ such that $T\llcorner\gamma$ is a normal current, and for some finite Borel measure $\eta$ over $C\left(\mathbb{R}^{+} ; E\right)$ representing $T\llcorner\gamma$ without cancelation of mass one has $\mu_{t}=e_{t \#} \eta$ for all $t \in \mathbb{R}^{+}$.

Proof. Since $q_{\#} \sigma$ is absolutely continuous with respect to some measure representing $T$ without cancelation of mass, then by Lemma A.18 there is a $\gamma \in L^{1}\left(E, m_{T}\right)$ such that for an $S \in \mathcal{M}_{1}(E)$ defined by (A.9) one has that $S$ is normal and $S=T\llcorner\gamma$. Defining $\varphi: C\left(\mathbb{R}^{+} ; E\right) \times \mathbb{R}^{+} \rightarrow C\left(\mathbb{R}^{+} ; E\right)$ by $\varphi(\theta, s)(t):=\theta(s+t)$, we have that $\eta:=\varphi_{\#} \sigma$ represents $S$ without cancelation of mass. Finally,

$$
e_{t \#} \eta=e_{t \#}\left(\varphi_{\#} \sigma\right)=\left(e_{t} \circ \varphi\right)_{\#} \sigma=\beta_{t \#} \sigma=\mu_{t}
$$

concluding the proof.

Note that the flow $\mu_{t}$ may be not unique as the following example shows.

Example 4.12. Let $E:=\mathbb{R}^{2}, \eta:=\delta_{\theta}$, where $\theta$ is the self-intersecting curve shown in figure 4.12. The parameterization of $\theta$ is such that the point $A$ of self-intersection is attained at two instants $t_{1}$ and $t_{2}$ with $t_{2}>t_{1}$. Let $\sigma_{i}:=\eta \otimes \delta_{t_{i}}, i=1,2$. Then $\mu:=\delta_{A}$ is transportable by $\eta$ both through $\sigma_{1}$ and through $\sigma_{2}$, but the flows $\mu_{t}$ generated by $\sigma_{1}$ and by $\sigma_{2}$ move the measure $\delta_{A}$ along different branches of the curve $\theta$, namely, in the first case $\mu_{t}=\delta_{A_{t}^{1}}$, while in the second case $\mu_{t}=\delta_{A_{t}^{2}}$ (see figure 4.12).

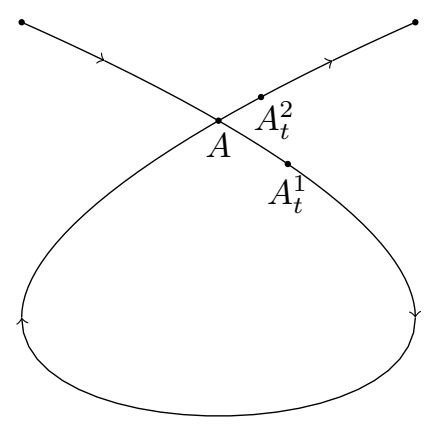

Figure 2. Nonuniqueness of the flow of $\delta_{A}$ in Example 4.12. 
Note that some (but of course not all) of the nonuniqueness of the flows is due to the fact, that the same curves may be parameterized by several different ways. In fact, if we reparameterize in some measurable way all the curves of in the support of the measure $\eta$ transporting the given measure $\mu$, we will get a new flow of the same measure. However, as the following easy statement shows, the measure $\mu$ will still remain transportable.

Proposition 4.13. Assume that $\eta$ be a $\sigma$-finite Borel measure on $C\left(\mathbb{R}^{+} ; E\right)$ and $\mu$ be a Borel measure over E transportable by $\eta$. Suppose that there exists a Borel reparameterization map

$$
\psi: C\left(\mathbb{R}^{+} ; E\right) \times \mathbb{R}^{+} \rightarrow \mathbb{R}^{+}
$$

such that $\psi(\theta, \cdot)$ is surjective, non decreasing and $\theta\left(t_{1}\right)=\theta\left(t_{2}\right)$ whenever $\psi\left(\theta, t_{1}\right)=$ $\psi\left(\theta, t_{2}\right)$. Then $\mu$ is transportable by $g_{\#} \eta$ where $g: C\left(\mathbb{R}^{+} ; E\right) \rightarrow C\left(\mathbb{R}^{+} ; E\right)$ is a Borel map uniquely defined by

$$
g(\theta)(\psi(\theta, t))=\theta(t) \quad \text { for all } t \in \mathbb{R}^{+} .
$$

Proof. Since $\psi(\theta, \cdot)$ is surjective, there exists a map $\psi^{-1}(\theta, \cdot)$ such that

$$
\psi\left(\theta, \psi^{-1}\left(\theta, t^{\prime}\right)\right)=t^{\prime}
$$

for all $t^{\prime} \in \mathbb{R}^{+}$. The map $\psi^{-1}(\theta, \cdot)$ must be strictly increasing and one can prove that $t^{\prime} \mapsto \theta\left(\psi^{-1}\left(t^{\prime}\right)\right)$ is continuous. Hence (4.4) is satisfied by a unique map $g: C\left(\mathbb{R}^{+} ; E\right) \rightarrow C\left(\mathbb{R}^{+} ; E\right)$ defined by $g(\theta)\left(t^{\prime}\right)=\theta\left(\psi^{-1}\left(\theta, t^{\prime}\right)\right)$ (note that although $\psi^{-1}$ clearly maybe non unique, the map $g$ is unique because $\theta\left(t_{1}\right)=\theta\left(t_{2}\right)$ whenever $\left.\psi\left(\theta, t_{1}\right)=\psi\left(\theta, t_{2}\right)\right)$.

We also define $f: C\left(\mathbb{R}^{+} ; E\right) \times \mathbb{R}^{+} \rightarrow C\left(\mathbb{R}^{+} ; E\right) \times \mathbb{R}^{+}$by $f(\theta, t)=(g(\theta), \psi(\theta, t))$, so that $\beta_{0} \circ f=\beta_{0}$ in view of (4.4). Moreover $q \circ f=g \circ q$ since $q(f(\theta, t))=g(\theta)=$ $g(q(\theta, t))$. If $\mu$ is transportable by $\eta$, then by Definition 4.1 there is a Borel measure $\sigma$ over $C\left(\mathbb{R}^{+} ; E\right) \times \mathbb{R}^{+}$satisfying (4.1). Setting $\sigma^{\prime}:=f_{\#} \sigma$ one has

$$
\beta_{0 \#} \sigma^{\prime}=\beta_{0 \#}\left(f_{\#} \sigma\right)=\left(\beta_{0} \circ f\right)_{\#} \sigma=\beta_{0 \#} \sigma=\mu
$$

and

$$
q_{\#} \sigma^{\prime}=q_{\#}\left(f_{\#} \sigma\right)=(q \circ f)_{\#} \sigma=(g \circ q)_{\#} \sigma=g_{\#}\left(q_{\#} \sigma\right) \ll g_{\#} \eta,
$$

which concludes the proof.

Remark 4.14. The most natural example of the situation of the above Proposition 4.13 is given by the arclength reparameterization of curves, i.e. $g(\theta)$ standing for the arclength reparameterization of $\theta$ and

$$
\psi(\theta, t):= \begin{cases}\ell(\theta\llcorner[0, t]) & \text { if } \ell(\theta\llcorner[0, t])<\ell(\theta) \\ \ell(\theta)+t-\bar{t}(\theta), & \text { otherwise, }\end{cases}
$$

where $\bar{t}(\theta):=\inf \{t: \ell(\theta\llcorner[0, t])=\ell(\theta)\}$, which is Borel in $\theta$ and continuous in $t$.

Remark 4.15. It is worth mentioning that always when $\eta$ represents some current $T$ without cancelation of mass, and a Borel map $g: C\left(\mathbb{R}^{+} ; E\right) \rightarrow C\left(\mathbb{R}^{+} ; E\right)$ is such that $g(\theta)$ is a reparameterization of $\theta$ for $\eta$-a.e. $\theta \in C\left(\mathbb{R}^{+} ; E\right)$, then the measure $g_{\#} \eta$ represents the same current and also without cancelation of mass.

In view of the above remarks the following immediate corollary to Proposition 4.13 holds true.

Corollary 4.16. Let $\mu$ be transportable by some laminated current $T \in \mathcal{M}_{1}(E)$. Then there is a $\sigma$-finite Borel measure $\eta$ representing $T$ without cancelation of mass and concentrated over arclength parameterized curves in $C\left(\mathbb{R}^{+} ; E\right)$ such that $\mu$ is transportable by $\eta$. 
A measure $\eta$ singled out in Corollary 4.16 is of course not unique (since the current $T$ may be represented by different measures over curves without cancelation of mass, and $\mu$ may be transportable by several of the latter); nevertheless in most situations such measures are the most interesting.

\section{REPRESENTATION OF CURRENTS AND VECTOR FIELDS}

The following result on representation of laminated currents (and hence also of measurable vector fields) will be important in the sequel.

Theorem 5.1. Let $E$ be a Banach space, $T \in \mathcal{M}_{1}(E)$ be a laminated current represented by a $\sigma$-finite Borel measure $\eta$ over $C\left(\mathbb{R}^{+} ; E\right)$ without cancelation of mass, such that $\eta$-a.e. $\theta$ is norm differentiable almost everywhere (in particular, this holds when E has Radon-Nikodym property since then all absolutely continuous curves are so). Then there is a Borel function $V_{T}: E \rightarrow E$ with $\left\|V_{T}(y)\right\|=1$ for $m_{T}$-a.e. $y \in E$ satisfying

$$
T(f d \pi)=\int_{E} f(y)\left\langle V_{T}(y), d \pi(y)\right\rangle d m_{T}(y),
$$

when $(f, \pi) \in L^{1}\left(E ; m_{T}\right) \times Q^{1}(E)$. Further, for $\eta$-a.e. $\theta \in C\left(\mathbb{R}^{+} ; E\right)$ and a.e. $t \in \mathbb{R}^{+}$ one has

$$
\dot{\theta}(t) \in V_{T}(\theta(t))^{\prime \prime}|\dot{\theta}|(t)
$$

(where $V_{T}^{\prime}$ is defined by (B.1)), and in particular, when the norm in E is strictly convex, then

$$
\dot{\theta}(t)=V_{T}(\theta(t))|\dot{\theta}|(t) .
$$

If, moreover, $\eta$-a.e. $\theta \in C\left(\mathbb{R}^{+} ; E\right)$ are parameterized by arclength, then for $\eta$-a.e. $\theta \in C\left(\mathbb{R}^{+} ; E\right)$ and a.e. $t \in[0, \ell(\theta))$ one has

$$
\dot{\theta}(t) \in V_{T}(\theta(t))^{\prime \prime},
$$

and in particular, when the norm in $E$ is strictly convex, then

$$
\dot{\theta}(t)=V_{T}(\theta(t)) \text {. }
$$

Moreover, the claims (5.2) and (5.4) hold for every $V_{T}$ satisfying (5.1).

Remark 5.2. If $E$ is a finite-dimensional normed space, then for every (not necessarily laminated) $T \in \mathcal{M}_{1}(E)$ a Borel map $V_{T}$ satisfying (5.1) exists by Lemma A.3. The representation (5.1) together with Proposition A.8 establishes the natural correspondence between one-dimensional metric currents in a Euclidean space, measurable vector fields and vector valued Borel maps. In fact, according to Proposition A.8 for every $T \in \mathcal{M}_{1}(E)$ there is a unique measurable vector field $X_{T}$ satisfying

$$
T(f d \pi)=\int_{E} f(y)\left(X_{T} \pi\right)(y) d m_{T}(y)
$$

for all $f d \pi \in D^{1}(E)$, and so $\left(X_{T} \pi\right)(y)=\left\langle V_{T}(y), d \pi(y)\right\rangle$ for $m_{T}$-a.e. $y \in E$. Clearly, if $T$ is normal, then additionally

$$
\operatorname{div}_{m_{T}} X_{T}=\operatorname{div} V_{T} m_{T}
$$

is a signed Borel measure of finite total variation (mind the different meanings of the two divergence symbols in the above relationship: it is the abstract divergence operator applied to measurable vector fields on the left-hand side and the usual distributional divergence on the right-hand side). Note that the map $V_{T}$ satisfying (5.1) is unique up to $m_{T}$-a.e. equality by Remark A.2. The above Theorem 5.1 gives therefore just the additional information on every representative of $V_{T}$ in the class of $m_{T}$-a.e. equal Borel maps in the case $T$ is laminated, or, equivalently, on the structure of measures over curves representing the laminated current $T$. 
Proof. We may further assume without loss of generality $E$ to be separable (substituting it, if necessary, with the linear span of $\operatorname{supp} m_{T}$ which is separable since $m_{T}$ is assumed to be tight). Let $\sigma$ be a finite measure over $C\left(\mathbb{R}^{+} ; E\right) \times \mathbb{R}^{+}$equivalent to $\eta \otimes \mathcal{L}^{1}$. Disintegrate the measure $\eta \otimes \mathcal{L}^{1}$ over $C\left(\mathbb{R}^{+} ; E\right) \times \mathbb{R}^{+}$as

$$
\eta \otimes \mathcal{L}^{1}=\mu \otimes \tau^{y}, \text { where } \mu:=\beta_{0 \#} \sigma,
$$

so that $\mu$ is equivalent to $\beta_{0 \#}\left(\eta \otimes \mathcal{L}^{1}\right)$, and $\tau^{y}$ are $\sigma$-finite measures concentrated over $\beta_{0}^{-1}(y) \subset C\left(\mathbb{R}^{+} ; E\right) \times \mathbb{R}^{+}$, and denote

$$
V(y):=\int_{C\left(\mathbb{R}^{+} ; E\right) \times \mathbb{R}^{+}} \dot{\theta}(t) d \tau^{y}(\theta, t)=\int_{\beta_{0}^{-1}(y)} \dot{\theta}(t) d \tau^{y}(\theta, t),
$$

the integrals of functions with values in $E$ here and below being intended in the sense of Bochner. Note that for $\mu$-a.e. $y \in E$ the measure $\tau^{y}$ does not charge $\eta \otimes \mathcal{L}^{1}$ nullsets, and thus for $\tau^{y}$-a.e. $(\theta, t)$ the derivative $\dot{\theta}(t)$ is well defined. Moreover, the $\operatorname{map}(\theta, t) \in C\left(\mathbb{R}^{+} ; E\right) \times \mathbb{R}^{+} \mapsto \dot{\theta}(t) \in E$ is $\eta \otimes \mathcal{L}^{1}$-a.e. equal to a Borel map by Lemma B.2, hence $\tau^{y}$-measurable, and so is also the map $(\theta, t) \in C\left(\mathbb{R}^{+} ; E\right) \times \mathbb{R}^{+} \mapsto$ $|\dot{\theta}|(t) \in E$ (say, because $|\dot{\theta}|(t)=\|\dot{\theta}(t)\|$ for a.e. $t \in \mathbb{R}^{+}$), and

$$
\begin{aligned}
\int_{E} d \mu(y) \int_{C\left(\mathbb{R}^{+} ; E\right) \times \mathbb{R}^{+}}|\dot{\theta}|(t) d \tau^{y}(\theta, t) & =\int_{\mathbb{R}^{+}} d t \int_{C\left(\mathbb{R}^{+} ; E\right)}|\dot{\theta}|(t) d \eta(\theta) \\
& =\int_{C\left(\mathbb{R}^{+} ; E\right)} \ell(\theta) d \eta(\theta)=\mathbb{M}(T),
\end{aligned}
$$

and hence $V(y)$ is well defined for $\mu$-a.e. $y \in E$.

Let $\pi \in Q^{1}(E)$. Observe that

$$
\begin{aligned}
T(f d \pi) & =\int_{C\left(\mathbb{R}^{+} ; E\right)} \llbracket \theta \rrbracket(f d \pi) d \eta(\theta) \\
& =\int_{C\left(\mathbb{R}^{+} ; E\right)} d \eta(\theta) \int_{\mathbb{R}^{+}} f(\theta(t))\langle\dot{\theta}(t), d \pi(\theta(t))\rangle d t .
\end{aligned}
$$

Recalling that $f \in C_{b}(E)$ and that $\|d \pi(y)\|^{\prime} \leq \operatorname{Lip} \pi$, we get

$$
\begin{aligned}
\int_{C\left(\mathbb{R}^{+} ; E\right)} d \eta(\theta) & \int_{\mathbb{R}^{+}}|f(\theta(t))\langle\dot{\theta}(t), d \pi(\theta(t))\rangle| d t \\
& \leq\|f\|_{\infty} \operatorname{Lip} \pi \int_{C\left(\mathbb{R}^{+} ; E\right)} d \eta(\theta) \int_{\mathbb{R}^{+}}|\dot{\theta}|(t) d t \\
& =\|f\|_{\infty} \operatorname{Lip} \pi \int_{C\left(\mathbb{R}^{+} ; E\right)} \ell(\theta) d \eta(\theta)=\|f\|_{\infty} \operatorname{Lip} \pi \mathbb{M}(T)<+\infty
\end{aligned}
$$

and thus we may change the order of integration in (5.8) obtaining

$$
\begin{aligned}
T(f d \pi) & =\int_{C\left(\mathbb{R}^{+} ; E\right) \times \mathbb{R}^{+}} f(\theta(t))\langle\dot{\theta}(t), d \pi(\theta(t))\rangle d\left(\eta \otimes \mathcal{L}^{1}\right)(\theta, t) \\
& =\int_{E} d \mu(y) \int_{C\left(\mathbb{R}^{+} ; E\right) \times \mathbb{R}^{+}} f(\theta(t))\langle\dot{\theta}(t), d \pi(\theta(t))\rangle d \tau^{y}(\theta, t) \\
& =\int_{E} f(y)\left\langle\left(\int_{C\left(\mathbb{R}^{+} ; E\right) \times \mathbb{R}^{+}} \dot{\theta}(t) d \tau^{y}(\theta, t)\right), d \pi(y)\right\rangle d \mu(y) \\
& =\int_{E} f(y)\langle V(y), d \pi(y)\rangle d \mu(y) .
\end{aligned}
$$

The latter also implies $m_{T}=\|V\| \mu$ by Lemma A.1. Denoting $V_{T}(y):=V(y) /\|V(y)\|$ with the convention $0 / 0:=0$, we note that $V_{T}$ may be assumed to be Borel up to 
changing it on a set of zero measure $m_{T}$, that $\left\|V_{T}(y)\right\|=1$ for $m_{T}$-a.e. $y \in E$ and (5.1) holds. Moreover,

$$
\|V(y)\|=\left\|\int_{C\left(\mathbb{R}^{+} ; E\right) \times \mathbb{R}^{+}} \dot{\theta}(t) d \tau^{y}(\theta, t)\right\|=\int_{C\left(\mathbb{R}^{+} ; E\right) \times \mathbb{R}^{+}}|\dot{\theta}|(t) d \tau^{y}(\theta, t),
$$

for $\mu$-a.e. $y \in E$, in view of (5.7) and the equality $\int_{E}\|V(y)\| d \mu(y)=\mathbb{M}(T)$. Therefore for such $y \in E$ one has

$$
\dot{\theta}(t) \in \frac{\left(\int_{C\left(\mathbb{R}^{+} ; E\right) \times \mathbb{R}^{+}} \dot{\theta}(s) d \tau^{y}(\theta, s)\right)^{\prime \prime}}{\int_{C\left(\mathbb{R}^{+} ; E\right) \times \mathbb{R}^{+}}|\dot{\theta}|(s) d \tau^{y}(\theta, s)}|\dot{\theta}|(t),
$$

by Lemma B.1 or, in other words,

$$
\dot{\theta}(t) \in \frac{V(y)^{\prime \prime}}{\|V(y)\|}|\dot{\theta}|(t)=V_{T}(y)^{\prime \prime}|\dot{\theta}|(t)
$$

for $\tau^{y}$-a.e. $(\theta, t) \in C\left(\mathbb{R}^{+} ; E\right) \times \mathbb{R}^{+}$, so that $\eta$-a.e. $\theta \in C\left(\mathbb{R}^{+} ; E\right)$ satisfies (5.2) for a.e. $t \in \mathbb{R}^{+}$. Note that (5.11) makes sense since for $\mu$-a.e. $y \in E$ such that $V(y)=0$ one has $|\dot{\theta}|(t)=0$ for $\tau^{y}$-a.e. $(\theta, t) \in C\left(\mathbb{R}^{+} ; E\right) \times \mathbb{R}^{+}$. Therefore, also

$$
\eta \otimes \mathcal{L}^{1}\left(\left\{(\theta, t) \in C\left(\mathbb{R}^{+} ; E\right) \times \mathbb{R}^{+}: V(\theta(t))=0 \text { but }|\dot{\theta}|(t) \neq 0\right\}\right)=0
$$

(where by writing $|\dot{\theta}|(t) \neq 0$ for brevity we mean that either $|\dot{\theta}|(t)$ is defined and nonzero, or is undefined). The relationships (5.3), (5.4) and (5.5) are now straightforward from (5.11).

It remains to show that (5.4) and (5.2) hold for every $V_{T}$ satisfying (5.1). We start with (5.4). Suppose that $T=\tilde{V}_{T} \wedge m_{T}$. Then $V_{T}(x)=\tilde{V}_{T}(x)$ for $m_{T}$-a.e. $x \in E$ by Remark A.2. We show that then for $\eta$-a.e. $\theta \in C\left(\mathbb{R}^{+} ; E\right)$ and a.e. $t \in[0, \ell(\theta))$ the relationship (5.4) is valid with $\tilde{V}_{T}$ instead of $V_{T}$. This is true since $m_{T}=\beta_{0 \#}\left(\eta \otimes \mathcal{L}^{1}\llcorner[0, \ell(\theta)))\right.$, and hence

$$
\begin{gathered}
\eta \otimes \mathcal{L}^{1}\left\llcorner[0, \ell(\theta))\left(\left\{(\theta, t) \in C\left(\mathbb{R}^{+} ; E\right) \times \mathbb{R}^{+}: V_{T}(\theta(t)) \neq \tilde{V}_{T}(\theta(t))\right\}\right)\right. \\
=m_{T}\left(\left\{x \in E: V_{T}(x) \neq \tilde{V}_{T}(x)\right\}\right)=0,
\end{gathered}
$$

proving the claim.

At last, we show that (5.2) holds for every $V_{T}$ satisfying (5.1). Let $g(\theta)$ stand for the arclength reparameterization of $\theta$, that is $g(\theta)(t):=\theta\left(\varphi_{\theta}(t \wedge \ell(\theta))\right)$, where $\varphi_{\theta}(s):=\inf \left\{t \in \mathbb{R}^{+}: \psi_{\theta}(t)=s\right\}, \psi_{\theta}(t):=\ell\left(\theta\llcorner[0, t])\right.$. Denoting $\eta^{\prime}:=g_{\#} \eta$, we get from (5.4) for $\eta^{\prime}$-a.e. $\gamma \in C\left(\mathbb{R}^{+} ; E\right)$ and a.e. $t \leq \ell(\gamma)$, the inclusion

$$
\dot{\gamma}(t) \in V_{T}(\gamma((t)))^{\prime \prime} .
$$

But since for $\gamma=g(\theta)$ one has

$$
\dot{\gamma}(t)=\dot{\theta}\left(\varphi_{\theta}(t)\right) \cdot \dot{\varphi}_{\theta}(t)=\frac{\dot{\theta}\left(\varphi_{\theta}(t)\right)}{|\dot{\theta}|\left(\varphi_{\theta}(t)\right)},
$$

for a.e. $t \leq \ell(\gamma)=\ell(\theta)$, we get from (5.12) the relationship

$$
\frac{\dot{\theta}\left(\varphi_{\theta}(t)\right)}{|\dot{\theta}|\left(\varphi_{\theta}(t)\right)} \in V_{T}\left(\theta\left(\varphi_{\theta}(t)\right)\right)^{\prime \prime} .
$$

This means that the set of $t \in \mathbb{R}^{+}$such that $\dot{\psi}_{\theta}(t)=|\dot{\theta}|(t) \neq 0$ and

$$
\frac{\dot{\theta}(t)}{|\dot{\theta}|(t)} \notin V_{T}(\theta(t))^{\prime \prime}
$$

has zero Lebesgue measure (in view of the area formula), which implies (5.2) for $\eta$-a.e. $\theta \in C\left(\mathbb{R}^{+} ; E\right)$ and a.e. $t \in \mathbb{R}^{+}$and hence concludes the proof for the case when $\eta$-a.e. $\theta \in C\left(\mathbb{R}^{+} ; E\right)$ are norm differentiable almost everywhere. 
It is not difficult to show that when the norm in $E$ is not strictly convex, then the inclusion in (5.2) (or (5.3)) does not necessarily become an equality, as the following easy though instructive example shows.

Example 5.3. Equip $E:=\mathbb{R}^{2}$ with the norm $\|\cdot\|_{1}$ defined by $\left\|\left(x_{1}, x_{2}\right)\right\|_{1}:=\left|x_{1}\right|+\left|x_{2}\right|$. Define $\eta_{i}, i=1,2$, by the formulae

$$
\begin{aligned}
& \eta_{1}:=\mathcal{L}^{1}\left\llcorner[0,1] \otimes \delta_{\theta_{x_{2}}}, \quad \text { where } \quad \theta_{x_{2}}(t):=\left(t \wedge 1, x_{2}\right) \quad \text { for each } x_{2} \in[0,1],\right. \\
& \eta_{2}:=\mathcal{L}^{1}\left\llcorner[0,1] \otimes \delta_{\theta_{x_{1}}}, \quad \text { where } \quad \theta_{x_{1}}(t):=\left(x_{1}, t \wedge 1\right) \quad \text { for each } x_{1} \in[0,1]\right.
\end{aligned}
$$

(with the parameter $t \in \mathbb{R}^{+}$). Clearly, $T_{\eta_{i}}=\bar{e}_{i} \wedge \mathcal{L}^{2}\left\llcorner([0,1] \times[0,1]), \mathbb{M}\left(T_{\eta_{i}}\right)=1\right.$. Setting $\eta:=\eta_{1}+\eta_{2}$, one has $T:=T_{\eta_{1}}+T_{\eta_{2}}=\left(\bar{e}_{1}+\bar{e}_{2}\right) \wedge \mathcal{L}^{2}\llcorner Q$, thus $\mathbb{M}(T)=2$, and hence $T_{\eta_{i}} \leq T$ and $\eta$ represents $T$ without cancelation of mass. One has $m_{T}=2 \mathcal{L}^{2}\left\llcorner Q, V_{T}=\left(\bar{e}_{1}+\bar{e}_{2}\right) / 2\right.$, and $\dot{\theta}(t) \in V_{T}^{\prime \prime}$ for $\eta$-a.e. $\theta$ and for all $t \in[0,1]$ (because $V_{T}^{\prime \prime}=\left\{\lambda_{1} \bar{e}_{1}+\lambda_{2} \bar{e}_{2}: \lambda_{i} \geq 0, i=1,2\right\}$ in this case), but $\dot{\theta}(t) \neq V_{T}$.

It is worth remarking however, that if one equips $E$ instead with the usual Euclidean norm, then one would still have $\mathbb{M}\left(T_{\eta_{i}}\right)=1$, but $\mathbb{M}(T)=\sqrt{2}$, hence $T_{\eta_{i}} \not \leq T$, $i=1,2$, and, moreover,

$$
\int_{C\left(\mathbb{R}^{+} ; E\right)} \mathbb{M}(\llbracket \theta \rrbracket) d \eta=2>\mathbb{M}(T),
$$

and thus $\eta$ does not represent $T$ without cancelation of mass.

A few remarks regarding the above Theorem 5.1 and its proof have to be made.

Remark 5.4. If $E$ is a Banach space and $\eta$ is just some $\sigma$-finite measure over $C\left(\mathbb{R}^{+} ; E\right)$ concentrated over absolutely continuous almost everywhere norm differentiable curves and one has

$$
\left\|\int_{C\left(\mathbb{R}^{+} ; E\right) \times \mathbb{R}^{+}} \dot{\theta}(t) d \tau^{y}(\theta, t)\right\|=\int_{C\left(\mathbb{R}^{+} ; E\right) \times \mathbb{R}^{+}}|\dot{\theta}|(t) d \tau^{y}(\theta, t)
$$

for $\mu$-a.e. $y \in E$, or, equivalently (by Lemma B.1), with the notation of the proof,

$$
\dot{\theta}(t) \in \frac{V(y)^{\prime \prime}}{\|V(y)\|}|\dot{\theta}|(t)
$$

for $\tau^{y}$-a.e. $(\theta, t) \in C\left(\mathbb{R}^{+} ; E\right) \times \mathbb{R}^{+}$and for $\mu$-a.e. $y \in E$, then $\eta$ represents $T_{\eta} \in$ $\mathcal{M}_{1}(E)$ without cancelation of mass. In fact, since

$$
T_{\eta}(f d \pi)=\int_{E} f(y)\langle V(y), d \pi(y)\rangle d \mu(y),
$$

where $\pi \in Q^{1}(E)$, by (5.9) then $\mathbb{M}\left(T_{\eta}\right)=\int_{E}\|V(y)\| d \mu(y)$ in view of Lemma A.1. Thus

$$
\begin{aligned}
\mathbb{M}\left(T_{\eta}\right) & =\int_{E} d \mu(y)\left\|\int_{C\left(\mathbb{R}^{+} ; E\right) \times \mathbb{R}^{+}} \dot{\theta}(t) d \tau^{y}(\theta, t)\right\| \\
& =\int_{E} d \mu(y) \int_{C\left(\mathbb{R}^{+} ; E\right) \times \mathbb{R}^{+}}|\dot{\theta}|(t) d \tau^{y}(\theta, t) \\
& =\int_{\mathbb{R}^{+}} d t \int_{C\left(\mathbb{R}^{+} ; E\right)}|\dot{\theta}|(t) d \eta(\theta)=\int_{C\left(\mathbb{R}^{+} ; E\right)} \ell(\theta) d \eta(\theta),
\end{aligned}
$$

showing the claim.

Remark 5.5. If under the conditions of the above Theorem 5.1 one has additionally that $\eta$ is finite (and thus the current $T$ is normal), and $\eta$-a.e. $\theta$ is parameterized by arclength, then

$$
V_{T}(\theta(t))=\mathbb{E}\left(\dot{\theta} ; \beta_{0}^{-1}(\mathscr{B}(E))\right)(t)
$$


where $\mathbb{E}(\cdot ; \Sigma)$ stands for the conditional expectation with respect to the $\sigma$-algebra $\Sigma$. To see this, one recalls that $\beta_{0 \#}\left(\eta \otimes \mathcal{L}^{1}\llcorner[0, \ell(\theta)])=m_{T}\right.$ (Example 4.5) and calculates

$$
\begin{aligned}
\int_{\beta_{0}^{-1}(e)} & \mathbb{E}\left(\dot{\theta} ; \beta_{0}^{-1}(\mathscr{B}(E))\right)(t) d\left(\eta \otimes \mathcal{L}^{1}\llcorner[0, \ell(\theta)])(\theta, t)\right. \\
& =\int_{E} d m_{T}(y) \int_{\beta_{0}^{-1}(y)} \mathbf{1}_{\beta_{0}^{-1}(B)}(\theta, t) \mathbb{E}\left(\dot{\theta} ; \beta_{0}^{-1}(\mathscr{B}(E))\right)(t) d \bar{\tau}^{y}(\theta, t) \\
& =\int_{E} \mathbf{1}_{B}(y) d m_{T}(y) \int_{\beta_{0}^{-1}(y)} \mathbb{E}\left(\dot{\theta} ; \beta_{0}^{-1}(\mathscr{B}(E))\right)(t) d \bar{\tau}^{y}(\theta, t) \\
& =\int_{B} d m_{T}(y) \int_{\beta_{0}^{-1}(y)} \mathbb{E}\left(\dot{\theta} ; \beta_{0}^{-1}(\mathscr{B}(E))\right)(t) d \bar{\tau}^{y}(\theta, t)
\end{aligned}
$$

for every $B \in \mathscr{B}(E)$, where $\bar{\tau}^{y}$ is a Borel probability measure concentrated over $\beta_{0}^{-1}(y)$ and satisfying the disintegration identity

$$
\eta \otimes \mathcal{L}^{1}\left\llcorner[0, \ell(\theta)]=m_{T} \otimes \bar{\tau}^{y} .\right.
$$

Keeping in mind that

$$
\mathbb{E}\left(\dot{\theta} ; \beta_{0}^{-1}(\mathscr{B}(E))\right)(t)=\lambda\left(\beta_{0}(\theta, t)\right)=\lambda(\theta(t))
$$

for some Borel vector function of $\lambda$ over $E$, we have that over $\beta_{0}^{-1}(y)$ the latter conditional expectation depends only on $y$ (and not on $\theta$ and $t$ ), so that

$$
\int_{\beta_{0}^{-1}(B)} \mathbb{E}\left(\dot{\theta} ; \beta_{0}^{-1}(\mathscr{B}(E))\right)(t) d\left(\eta \otimes \mathcal{L}^{1}\llcorner[0, \ell(\theta)])=\int_{B} \lambda(y) d m_{T}(y) .\right.
$$

But on the other hand, by definition of the conditional expectation one has

$$
\begin{aligned}
\int_{\beta_{0}^{-1}(B)} & \mathbb{E}\left(\dot{\theta} ; \beta_{0}^{-1}(\mathscr{B}(E))\right)(t) d\left(\eta \otimes \mathcal{L}^{1}\llcorner[0, \ell(\theta)])(\theta, t)\right. \\
& =\int_{B} d m_{T}(y) \int_{\beta_{0}^{-1}(y)} \dot{\theta}(t) d \bar{\tau}^{y}(\theta, t)=\int_{B} V_{T}(y) d m_{T}(y),
\end{aligned}
$$

recalling (5.5). Thus $\lambda(y)=V_{T}(y)$ for $m_{T}$-a.e. $y \in E$, which is exactly the claim being proven.

Remark 5.6. It is important to remark that when $\eta$-a.e. $\theta \in C\left(\mathbb{R}^{+} ; E\right)$ is parameterized by arclength, then one can assert (5.4) (or, in a particular case, (5.5)) for $\eta$-a.e. $\theta \in C\left(\mathbb{R}^{+} ; E\right)$ only for a.e. $t \in[0, \ell(\theta))$ but not for a.e. $t \in \mathbb{R}^{+}$as the following simple example shows.

Example 5.7. Let $E:=\mathbb{R}^{2}$ and $\eta:=\mathcal{L}^{1}\left\llcorner[0,2] \otimes \delta_{\theta_{y}}+\delta_{\bar{\theta}}\right.$, where $\theta_{y}(t):=(t \wedge 1, y)$ for each $y \in[0,2]$ and $\bar{\theta}(t):=(1, t \wedge 2)$ (with the parameter $t \in \mathbb{R}^{+}$). This measure represents the current $\left(\mathcal{L}^{2}\llcorner[0,1] \times[0,2]) \wedge \bar{e}_{1}+\left(\mathcal{H}^{1}\llcorner I) \wedge \bar{e}_{2}\right.\right.$ without cancelation of mass, where $I$ stands for the vertical segment with endpoints $(1,0)$ and $(1,2)$ (see figure 5.7). Clearly, one has $V_{T}(y)=\bar{e}_{1}$ for $\mathcal{L}^{2}$-a.e. $y \in(0,1) \times(0,2)$ and $V_{T}(y)=\bar{e}_{2}$ for $\mathcal{H}^{1}$-a.e. $y \in I$. Thus, say, for all $y \in(1,2]$ one has $\dot{\theta}_{y}(t)=0$ for all $t \in(1,2)$, but $V_{T}\left(\theta_{y}(t)\right)=V_{T}((1, y))=\bar{e}_{2} \neq 0$ for $\mathcal{L}^{1}$-a.e. $y \in(1,2]$, and the measure $\eta$ of this set of curves is positive.

\section{Flows generated By measurable VeCtor Fields}

The following theorem gives the characterization of flows of a measure transportable by some measurable vector field (or, equivalently, one-dimensional current) in a Banach space. 


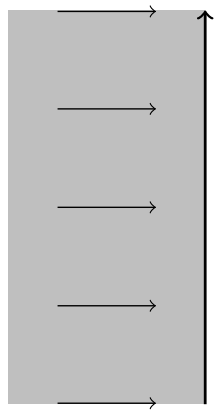

FIgURE 3. The situation in Example 5.7

Theorem 6.1. Assume a finite measure $\mu$ over a Banach space $E$ be transportable by a $\sigma$-finite measure $\eta$ concentrated over some separable subset of $C\left(\mathbb{R}^{+} ; E\right)$ consisting of only absolutely continuous curves, through a measure $\sigma$ over $C\left(\mathbb{R}^{+} ; E\right) \times \mathbb{R}^{+}$ such that $\tilde{\eta}:=q_{\#} \sigma$ satisfies

$$
\int_{C\left(\mathbb{R}^{+} ; E\right)} \ell(\theta) d \tilde{\eta}(\theta)<+\infty,
$$

and $\eta$-a.e. $\theta$ is norm differentiable almost everywhere (in particular, the latter holds when $E$ has Radon-Nikodym property since then all absolutely continuous curves are so). Then the following assertions are valid.

(A) Representing by disintegration $\sigma=\mu_{t} \otimes \nu_{t}^{y}$, where $\mu_{t}:=\beta_{t \#} \sigma$, so that each $\nu_{t}^{y}$ is a probability measure concentrated over

$$
\beta_{t}^{-1}(y)=\left\{(\theta, s) \in C\left(\mathbb{R}^{+} ; E\right) \times \mathbb{R}^{+}: \theta(s+t)=y\right\},
$$

and defining

$$
v_{t}(y):=\int_{C\left(\mathbb{R}^{+} ; E\right) \times \mathbb{R}^{+}} \dot{\theta}(s+t) d \nu_{t}^{y}(\theta, s)=\int_{\beta_{t}^{-1}(y)} \dot{\theta}(s+t) d \nu_{t}^{y}(\theta, s),
$$

we have that $v_{t}$ is Bochner-integrable with respect to $\mu_{t}$ for a.e. $t \in \mathbb{R}^{+}$ (and even more, the function $v: E \times \mathbb{R}^{+} \rightarrow E$ defined by $v(t, x):=v_{t}(x)$ is Bochner integrable with respect to $\left.d t \otimes \mu_{t}\right)$, and the continuity equation

$$
\frac{\partial \mu_{t}}{\partial t}+\operatorname{div} v_{t} \mu_{t}=0
$$

is valid in the weak sense, i.e.

$$
-\int_{\mathbb{R}^{+}} \dot{\psi}(t) d t \int_{E} \varphi(y) d \mu_{t}(y)-\int_{\mathbb{R}^{+}} \psi(t) d t \int_{E}\left\langle v_{t}(y), d \varphi(y)\right\rangle d \mu_{t}(y)=0
$$

for every $\varphi \in Q^{1}(E) \cap C_{b}(E)$ and $\psi \in C_{0}^{1}\left(\mathbb{R}^{+}\right)$.

(B) If, moreover, $\eta$ represents some laminated current $T \in \mathcal{M}_{1}(E)$ without cancelation of mass, then Theorem 5.1 holds. In particular, if in this case $\eta$-a.e. $\theta \in C\left(\mathbb{R}^{+} ; E\right)$ is parameterized by arclength, then

$$
\dot{\theta}(t) \in V_{T}(\theta(t))^{\prime \prime}
$$

(with the equality $\dot{\theta}(t)=V_{T}(\theta(t))$ if $E$ is strictly convex) for $\eta$-a.e. $\theta \in$ $C\left(\mathbb{R}^{+} ; E\right)$ and for a.e. $t \in[0, \ell(\theta))$. If in addition $E$ is strictly convex, then one has also

$$
v_{t}(y)=V_{T}(y)\left(1-\varphi_{t}(y)\right)
$$


for a.e. $t \in \mathbb{R}^{+}$and $\mu_{t}$-a.e. $y \in E$, where $\varphi_{t}(y):=\nu_{t}^{y}\left(S_{t}^{y}\right)$ stands for the proportion of mass which is stopped at point $y$ after time $t$,

$$
\begin{aligned}
S_{t}^{y} & :=\left\{(\theta, s) \in C\left(\mathbb{R}^{+} ; E\right) \times \mathbb{R}^{+}: \theta(s+t)=y, s+t \geq \ell(\theta)\right\} \\
& =\left\{(\theta, s) \in C\left(\mathbb{R}^{+} ; E\right) \times \mathbb{R}^{+}: \theta(s+t)=y=\operatorname{end}(\theta)\right\},
\end{aligned}
$$

and thus, in particular,

$$
\frac{\partial \mu_{t}}{\partial t}+\operatorname{div} V_{T}\left(1-\varphi_{t}\right) \mu_{t}=0
$$

in the weak sense.

It is worth remarking that the construction (6.2) of the vector field $v_{t}$ as the superposition of the speeds of the relevant curves is quite similar to the construction of $\left[17\right.$, theorem 7]. Note that the term containing $\varphi_{t}$ must appear in (6.7) in view of the Remark 5.6. It is also worth emphasizing that the relationship (6.6) is just a $\mu_{t}$-a.e. equality. For instance, in Example 5.7 with $\mu:=\mathcal{H}^{1}\left\llcorner(\{0\} \times[0,2])+\delta_{(1,0)}\right.$ one gets $\mu_{t}=\mathcal{H}^{1}\left\llcorner(\{t \wedge 1\} \times[0,2])+\delta_{(1, t \wedge 2)}\right.$, and, when $t>1$, one has $v_{t}(x)=\bar{e}_{2}$ for $x=(1, t \wedge 2)$ and $v_{t}(x)=0$ elsewhere.

Proof. We divide the proof in several steps in order to simplify the reading.

Step 1. First we show that (6.2) is a correct definition. To this aim let $N_{\theta} \in \mathbb{R}^{+}$ for a given $\theta \in C\left(\mathbb{R}^{+} ; E\right)$ stand for the set

$$
N_{\theta}:=\left\{s \in \mathbb{R}^{+}: \dot{\theta}(s) \text { is undefined }\right\} .
$$

Representing by disintegration $\sigma=\tilde{\eta} \otimes \lambda_{\theta}$ with $\lambda_{\theta}$ Borel probability measures over $\mathbb{R}^{+}$, we get from Lemma 6.2 (applied with $\left.\mu:=\lambda_{\theta}, B:=N_{\theta}\right)$ that $\lambda_{\theta}\left(N_{\theta}-t\right)=0$ for a.e. $t \in \mathbb{R}$ and $\tilde{\eta}$-a.e. $\theta \in C\left(\mathbb{R}^{+} ; E\right)$. Hence, observing that

$$
N_{\theta}-t=\left\{s \in \mathbb{R}^{+}: \dot{\theta}(s+t) \text { is undefined }\right\},
$$

we get that

$$
\sigma\left(\left\{(\theta, s) \in C\left(\mathbb{R}^{+} ; E\right) \times \mathbb{R}^{+}: \dot{\theta}(s+t) \text { is undefined }\right\}\right)=0
$$

for a.e. $t \in \mathbb{R}^{+}$, which implies that for such $t \in \mathbb{R}^{+}$the derivative $\dot{\theta}(s+t)$ is well defined for $\nu_{t}^{y}$-a.e. $(\theta, s) \in C\left(\mathbb{R}^{+} ; E\right) \times \mathbb{R}^{+}$and $\mu_{t}$-a.e. $y \in E$. Further, the function $(\theta, s) \in C\left(\mathbb{R}^{+} ; E\right) \times \mathbb{R}^{+} \mapsto \dot{\theta}(s+t)$ is $\tilde{\eta} \otimes \mathcal{L}^{1}$-a.e. equal to a Borel map by Lemma B.2, hence $\nu_{t}^{y}$-measurable (and automatically separable valued by an assumption on $\eta$ ).

Now the estimate

$$
\begin{aligned}
\int_{\mathbb{R}^{+}} & d t \int_{E} d \mu_{t}(y)\left(\int_{C\left(\mathbb{R}^{+} ; E\right) \times \mathbb{R}^{+}}|\dot{\theta}|(s+t) d \nu_{t}^{y}(\theta, s)\right) \\
= & \int_{C\left(\mathbb{R}^{+} ; E\right) \times \mathbb{R}^{+}} d \sigma(\theta, s) \int_{\mathbb{R}^{+}}|\dot{\theta}|(s+t) d t \\
= & \int_{C\left(\mathbb{R}^{+} ; E\right) \times \mathbb{R}^{+}} d \sigma(\theta, s) \int_{s}^{+\infty}|\dot{\theta}|(\tau) d \tau \\
= & \int_{C\left(\mathbb{R}^{+} ; E\right) \times \mathbb{R}^{+}} \ell\left(\theta\llcorner[s,+\infty)) d \sigma(\theta, s) \leq \int_{C\left(\mathbb{R}^{+} ; E\right) \times \mathbb{R}^{+}} \ell(\theta) d \sigma(\theta, s)\right. \\
= & \int_{C\left(\mathbb{R}^{+} ; E\right)} \ell(\theta) d \tilde{\eta}(\theta)<+\infty
\end{aligned}
$$

shows that the Bochner integral in (6.2) is well defined for a.e. $t \in \mathbb{R}^{+}$and $\mu_{t}$-a.e. $y \in E$, and, moreover, that for such $t$ one has $v_{t}$ is Bochner-integrable with respect 
to $\mu_{t}$ for a.e. $t \in \mathbb{R}^{+}$(and even more, the function $v: E \times \mathbb{R}^{+} \rightarrow E$ defined by $v(t, x):=v_{t}(x)$ is Bochner integrable with respect to $\left.d t \otimes \mu_{t}\right)$, because

$$
\begin{aligned}
\int_{\mathbb{R}^{+}} d t & \int_{E}\left\|v_{t}(y)\right\| d \mu_{t}(y) \\
& \leq \int_{\mathbb{R}^{+}} d t \int_{E} d \mu_{t}(y)\left(\int_{C\left(\mathbb{R}^{+} ; E\right) \times \mathbb{R}^{+}}|\dot{\theta}|(s+t) d \nu_{t}^{y}(\theta, s)\right)<+\infty .
\end{aligned}
$$

Step 2. We show now the validity of the continuity equation (6.3). Let $\varphi \in$ $Q^{1}(E) \cap C_{b}(E)$. Since, in the notation of Step 1, for $\tilde{\eta}$-a.e. $\theta \in C\left(\mathbb{R}^{+} ; E\right)$ the equality $\frac{d}{d t} \varphi(\theta(t))=\langle\dot{\theta}(t), d \varphi(\theta(t))\rangle$ is valid for all $t \in \mathbb{R}^{+} \backslash \tilde{N}_{\theta}$ where $\mathcal{L}^{1}\left(\tilde{N}_{\theta}\right)=0$, then from Lemma 6.2 (applied with $\left.\mu:=\lambda_{\theta}, B:=\tilde{N}_{\theta}\right)$ we have that $\lambda_{\theta}\left(\tilde{N}_{\theta}-t\right)=0$ for a.e. $t \in \mathbb{R}^{+}$and $\tilde{\eta}$-a.e. $\theta \in C\left(\mathbb{R}^{+} ; E\right)$, and thus

$$
\sigma\left(\left\{(\theta, s) \in C\left(\mathbb{R}^{+} ; E\right) \times \mathbb{R}^{+}: \frac{d}{d t} \varphi(\theta(s+t)) \neq\langle\dot{\theta}(s+t), d \varphi(\theta(s+t))\rangle\right\}\right)=0
$$

for a.e. $t \in \mathbb{R}^{+}$. Hence, for all such $t \in \mathbb{R}^{+}$for $\mu_{t}$-a.e. $y \in E$ one has

$$
\nu_{t}^{y}\left(\left\{(\theta, s) \in C\left(\mathbb{R}^{+} ; E\right) \times \mathbb{R}^{+}: \frac{d}{d t} \varphi(\theta(s+t)) \neq\langle\dot{\theta}(s+t), d \varphi(\theta(s+t))\rangle\right\}\right)=0,
$$

which implies

$$
\begin{aligned}
\int_{C\left(\mathbb{R}^{+} ; E\right) \times \mathbb{R}^{+}} & \frac{d}{d t} \varphi(\theta(s+t)) d \sigma(\theta, s) \\
= & \int_{E} d \mu_{t}(y)\left(\int_{C\left(\mathbb{R}^{+} ; E\right) \times \mathbb{R}^{+}} \frac{d}{d t} \varphi(\theta(s+t)) d \nu_{t}^{y}(\theta, s)\right) \\
= & \int_{E} d \mu_{t}(y)\left(\int_{C\left(\mathbb{R}^{+} ; E\right) \times \mathbb{R}^{+}}\langle\dot{\theta}(s+t), d \varphi(\theta(s+t))\rangle d \nu_{t}^{y}(\theta, s)\right) \\
= & \int_{E} d \mu_{t}(y)\left\langle\left(\int_{C\left(\mathbb{R}^{+} ; E\right) \times \mathbb{R}^{+}} \dot{\theta}(s+t) d \nu_{t}^{y}(\theta, s)\right), d \varphi(y)\right\rangle \\
= & \int_{E} d \mu_{t}(y)\left\langle v_{t}(y), d \varphi(y)\right\rangle .
\end{aligned}
$$

Note that the above calculation makes sense since $\left|\frac{d}{d t} \varphi(\theta(s+t))\right| \leq \operatorname{Lip} \varphi|\dot{\theta}|(s+t)$, so that the above integral is defined in view of (6.8). Multiplying the above equation by $\psi(t)$, integrating over $\mathbb{R}^{+}$and using integration by parts in $d t$, we get

$$
\begin{aligned}
\int_{\mathbb{R}^{+}} \psi(t) d t \int_{E}\left\langle v_{t}(y), d \varphi(y)\right\rangle d \mu_{t}(y) & =\int_{\mathbb{R}^{+}} \psi(t) d t \int_{C\left(\mathbb{R}^{+} ; E\right) \times \mathbb{R}^{+}} \frac{d}{d t} \varphi(\theta(s+t)) d \sigma(\theta, s) \\
& =\int_{C\left(\mathbb{R}^{+} ; E\right) \times \mathbb{R}^{+}} d \sigma(\theta, s) \int_{\mathbb{R}^{+}} \psi(t) \frac{d}{d t} \varphi(\theta(s+t)) d t \\
& =-\int_{C\left(\mathbb{R}^{+} ; E\right) \times \mathbb{R}^{+}} d \sigma(\theta, s) \int_{\mathbb{R}^{+}} \dot{\psi}(t) \varphi(\theta(s+t)) d t \\
& =-\int_{\mathbb{R}^{+}} \dot{\psi}(t) d t \int_{C\left(\mathbb{R}^{+} ; E\right) \times \mathbb{R}^{+}} \varphi(\theta(s+t)) d \sigma(\theta, s) \\
& =-\int_{\mathbb{R}^{+}} \dot{\psi}(t) d t \int_{E} \varphi(x) d \mu_{t}(y),
\end{aligned}
$$

so (6.3) (in the sense of $(6.4)$ ) is proven.

Step 3. Assume now that $\eta$ represent some laminated current $T$ without cancelation of mass. The relationship (6.5) follows from Theorem 5.1. Under the additional assumption that $\eta$-a.e. $\theta \in C\left(\mathbb{R}^{+} ; E\right)$ is parameterized by arclength, we have $\dot{\theta}(t) \in V_{T}(\theta(t))^{\prime \prime}$, and, if $E$ is also strictly convex, then $\dot{\theta}(t)=V_{T}(\theta(t))$ for 
$\eta$-a.e. $\theta \in C\left(\mathbb{R}^{+} ; E\right)$ and for a.e. $t \in[0, \ell(\theta))$ by Theorem 5.1. In other words, in the latter case the set

$$
M:=\left\{(\theta, \tau) \in C\left(\mathbb{R}^{+} ; E\right) \times \mathbb{R}^{+}: \dot{\theta}(\tau) \neq V_{T}(\theta(\tau)), \tau<\ell(\theta)\right\}
$$

satisfies $\left(\eta \otimes \mathcal{L}^{1}\right)(M)=0$. Setting $M_{\theta}:=\left\{\tau \in \mathbb{R}^{+}:(\theta, \tau) \in M\right\}$, we have $\mathcal{L}^{1}\left(M_{\theta}\right)=0$ for $\eta$-a.e. $\theta \in C\left(\mathbb{R}^{+} ; E\right)$. From Lemma 6.2 applied with $\mu:=\lambda_{\theta}$, $B:=M_{\theta}$ we conclude that $\lambda_{\theta}\left(M_{\theta}-t\right)=0$ for a.e. $t \in \mathbb{R}^{+}$and $\eta$-a.e. $\theta \in C\left(\mathbb{R}^{+} ; E\right)$. Hence, observing that

$$
M_{\theta}-t=\left\{s \in \mathbb{R}^{+}: \dot{\theta}(s+t) \neq V_{T}(\theta(s+t)), s+t<\ell(\theta)\right\},
$$

we obtain

$$
\sigma\left(\left\{(\theta, s) \in C\left(\mathbb{R}^{+} ; E\right) \times \mathbb{R}^{+}: \dot{\theta}(s+t) \neq V_{T}(\theta(s+t)), s+t<\ell(\theta)\right\}\right)=0
$$

for a.e. $t \in \mathbb{R}^{+}$, because $\sigma=\tilde{\eta} \otimes \lambda_{\theta}$ with $\tilde{\eta} \ll \eta$. Now (6.9) implies that $\dot{\theta}(s+t)=$ $V_{T}(\theta(s+t))$ for $\nu_{t}^{y}$-a.e. $(\theta, s) \in C\left(\mathbb{R}^{+} ; E\right) \times \mathbb{R}^{+}$such that $s+t<\ell(\theta)$ and $\mu_{t}$-a.e. $y \in E$, for a.e. $t \in \mathbb{R}^{+}$. Thus from (6.2) we get

$$
\begin{aligned}
v_{t}(y) & =\int_{\left(S_{t}^{y}\right)^{c}} \dot{\theta}(s+t) d \nu_{t}^{y}(\theta, s)=\int_{\left(S_{t}^{y}\right)^{c}} V_{T}(\theta(s+t)) d \nu_{t}^{y}(\theta, s) \\
& =\int_{\beta_{t}^{-1}(y) \backslash S_{t}^{y}} V_{T}(y) d \nu_{t}^{y}(\theta, s)=V_{T}(y)\left(1-\varphi_{t}(y)\right),
\end{aligned}
$$

which shows (6.6) and hence concludes the proof.

Lemma 6.2. Let $B \subset \mathbb{R}$ be a Borel set with $\mathcal{L}^{1}(B)=0$, and $\mu$ be a finite Borel measure over $\mathbb{R}$. Then $\mu(B-t)=0$ for a.e. $t \in \mathbb{R}$.

Proof. Otherwise there is a Borel set $\Delta \subset \mathbb{R}$ with $\mathcal{L}^{1}(\Delta)>0$ such that $\mu(B-t)>0$ for all $t \in \Delta$. This implies

$$
I:=\int_{\mathbb{R}} \mathbf{1}_{\Delta}(t) d t \int_{\mathbb{R}} \mathbf{1}_{B}(x+t) d \mu(x)>0,
$$

but on the other hand by Tonelli theorem on has

$$
I=\int_{\mathbb{R}} d \mu(x) \int_{\mathbb{R}} \mathbf{1}_{\Delta}(t) \mathbf{1}_{B}(x+t) d t=\int_{\mathbb{R}} d \mu(x) \int_{\mathbb{R}} \mathbf{1}_{\Delta}(t) \mathbf{1}_{B-x}(t) d t=0
$$

(since the inner integral vanishes), and this contradiction concludes the proof.

Remark 6.3. It is worth mentioning that under the conditions of Theorem 6.1(B) if $\eta$-a.e. $\theta \in C\left(\mathbb{R}^{+} ; E\right)$ is parameterized by arclength, then $\left\|v_{t}\right\| \mu_{t} \ll m_{T}$ for a.e. $t \in \mathbb{R}^{+}$. In fact, if $B \subset E$ is such that $m_{T}(B)=0$, then $\left(\eta \otimes \mathcal{L}^{1}\right)(\tilde{M})=0$, where

$$
\tilde{M}:=\left\{(\theta, \tau) \in C\left(\mathbb{R}^{+} ; E\right) \times \mathbb{R}^{+}: \theta(\tau) \in B, \tau<\ell(\theta) .\right\}
$$

Thus $\mathcal{L}^{1}\left(\tilde{M}_{\theta}\right)=0$, where $\tilde{M}_{\theta}:=\left\{\tau \in \mathbb{R}^{+}:(\theta, \tau) \in M\right\}$, for $\eta$-a.e. $\theta \in C\left(\mathbb{R}^{+} ; E\right)$, and hence by Lemma 6.2 (applied with $\lambda_{\theta}, \tilde{M}_{\theta}$ instead of $\mu, B$ respectively) one has $\lambda_{\theta}\left(\tilde{M}_{\theta}-t\right)=0$ for a.e. $t \in \mathbb{R}^{+}$and $\eta$-a.e. $\theta \in C\left(\mathbb{R}^{+} ; E\right)$, so that

$$
\sigma\left(\left\{(\theta, s) \in C\left(\mathbb{R}^{+} ; E\right) \times \mathbb{R}^{+}: \theta(s+t) \in B, s+t<\ell(\theta)\right\}\right)=0
$$

for a.e. $t \in \mathbb{R}^{+}$, because $\sigma=\tilde{\eta} \otimes \lambda_{\theta}$ with $\tilde{\eta} \ll \eta$. Now,

$$
\begin{aligned}
\int_{B}\left\|v_{t}(y)\right\| d \mu_{t}(y) & \leq \int_{B} d \mu_{t}(y)\left(\int_{C\left(\mathbb{R}^{+} ; E\right) \times \mathbb{R}^{+}}|\dot{\theta}|(s+t) d \nu_{t}^{y}(\theta, s)\right) \\
& =\int_{B} d \mu_{t}(y)\left(\int_{\left\{(\theta, s) \in C\left(\mathbb{R}^{+} ; E\right) \times \mathbb{R}^{+}: s+t<\ell(\theta)\right\}} d \nu_{t}^{y}(\theta, s)\right) \\
& =\sigma\left(\left\{(\theta, s) \in C\left(\mathbb{R}^{+} ; E\right) \times \mathbb{R}^{+}: \theta(s+t) \in B, s+t<\ell(\theta)\right\}\right)=0
\end{aligned}
$$

for a.e. $t \in \mathbb{R}^{+}$. 
One has to note that if $E$ is not strictly convex, then our construction may provide a lot of "unreasonable" flows of a given measure. For instance, in the notation of the Example 5.3 if $\mu:=\mathcal{H}^{1}\llcorner([0,1] \times\{0\})$, then the flow of $\mu$ produced by the measure $\eta$ (through $\sigma:=\eta \otimes \delta_{0}$, see Example 4.4) is given by $\mu_{t}:=\mathcal{H}^{1}\llcorner([0,1] \times\{t \wedge 1\})$, although $T=V_{T} \wedge m_{T}$ with $m_{T}=2 \mathcal{L}^{2}\left\llcorner Q, V_{T}=\left(\bar{e}_{1}+\bar{e}_{2}\right) / 2\right.$, i.e. this flow is not in the direction of $V_{T}$. This does not happen when we equip $E$ with a strictly convex (say, Euclidean) norm.

\section{CANONICAL FLOWS}

We present here two particular constructions of the flow which may be considered canonical.

7.1. Acyclic vector fields. Consider a measurable vector field corresponding to an acyclic normal current $T \in \mathcal{M}_{1}(E)$ (i.e. such that $C \in \mathcal{M}_{1}(E), C \leq T, \partial C=0$ implies $C=0)$. In this case there is a finite measure $\eta$ over $C\left(\mathbb{R}^{+} ; E\right)$ representing $T$ without cancelation of mass, with $(\partial T)^{+}=\operatorname{end}_{\#} \eta$ and $(\partial T)^{-}=\operatorname{start}_{\#} \eta$. We may claim now the following result.

Proposition 7.1. Let $\mu$ be a finite measure over $E$ with $\mu \perp(\partial T)^{+}$. The following assertions hold true.

(i) If $\mu$ is transportable by $\eta$, then there is a normal current $S \leq T$ with $\mu$ equivalent to $(\partial S)^{-}$. Further, one has that $\mu_{t}$ is equivalent to $e_{t \#} \bar{\eta}$ for some $\bar{\eta}$ representing $S$ without cancelation of mass.

(ii) If $\eta$ is concentrated over curves parameterized by arclength (which can always be assumed without loss of generality) and a.e. differentiable, $E$ is a strictly convex Banach space, then for any flow $\mu_{t}$ corresponding to $\eta$, if there is an interval $(0, \tau)$ such that $\mu_{t} \perp(\partial T)^{+}=0$ for $t \in(0, \tau)$, one has

$$
\frac{\partial \mu_{t}}{\partial t}+\operatorname{div} V_{T} \mu_{t}=0
$$

in the weak sense over $(0, \tau)$.

Proof. To prove (i), recall that by Remark 4.8, if $\mu$ is transportable by $\eta$, then there is a normal current $S \leq T$ such that $\partial S=\nu-\tilde{\mu}$ with $\nu$ equivalent to end $\# \eta$ and $\tilde{\mu}$ equivalent to $\mu$. But by assumption on $\eta$ one has $\operatorname{end}_{\#} \eta=(\partial T)^{+}$, hence $\mu \perp$ end $_{\#} \eta$ (by the assumption on $\mu$ ), and therefore, also $\tilde{\mu} \perp \operatorname{end}_{\#} \eta$. Hence $(\partial S)^{-}=\tilde{\mu}$. Further, in the notation of the same Remark 4.8 we have that $S$ is represented by a measure $\bar{\eta}$ over $C\left(\mathbb{R}^{+} ; E\right)$ without cancelation of mass, where

$$
\bar{\eta}(B):=\tilde{\sigma}\left(\left\{(\theta, s) \subset C\left(\mathbb{R}^{+} ; E\right) \times \mathbb{R}^{+}: s \in \mathbb{R}^{+}, r_{s}(\theta) \in B\right\}\right),
$$

where $r_{s}(\theta)(t)=e_{t}(r(\theta, s))=\theta(t+s)$, where $r: C\left(\mathbb{R}^{+} ; E\right) \times \mathbb{R}^{+} \rightarrow C\left(\mathbb{R}^{+} ; E\right)$ is defined by $r(\theta, s)(t):=\theta(t+s)$, and $B \subset C\left(\mathbb{R}^{+} ; E\right)$ is an arbitrary Borel set (in other words, $\left.\bar{\eta}:=r_{\#} \tilde{\sigma}\right)$. One has thus $\beta_{t \#} \tilde{\sigma}=e_{t \#} \bar{\eta}$ and recalling that $\mu_{t}$ is equivalent to $\beta_{t \# \tilde{\sigma}}$, one concludes the proof of (i).

Now, under the assumption of (ii) we have that $\mu_{t} \perp$ end $_{\#} \eta$ for all $t \leq \tau$. Hence, for $\mu_{t}$-a.e. $y \in E$ one has, in the notation of Theorem $6.1(\mathrm{C})$, that $S_{t}^{y}=\emptyset$, which gives $\varphi_{t}(y)=0$ for $t \leq \tau$, which proves (ii).

7.2. Cyclic vector fields. Consider a flow of a given measure generated by some cyclic, or, in other words, divergence free vector field, i.e. such that the respective current $T \in \mathcal{M}_{1}(E)$ is a cycle, $\partial T=0$. According to our definition this means a flow produced by some measures $\eta$ over $C\left(\mathbb{R}^{+} ; E\right)$ representing $T$ without cancelation of mass and concentrated over a set of curves of finite length. This might lead to a non-natural notion of the flow. For instance, if $\eta$-a.e. $\theta \in C\left(\mathbb{R}^{+} ; E\right)$ is parameterized by arclength, then $|\dot{\theta}|(t)=0$ for a $t>\ell(\theta)$, which means that each "particle" moving along the path $\theta$ stops at instant $\ell(\theta)$. Let, for instance, $T$ be a cycle associated to 
the clockwise oriented unit circumference in $E=\mathbb{R}^{2}$, let $\mu:=\delta_{(-1,0)}$, and observe that $T=T_{\eta}$ with $\eta:=\delta_{\theta_{1}}+\delta_{\theta_{2}}, \theta_{1}$ and $\theta_{2}$ being arclength parameterized right and left semi-circumferences, directed from top to bottom and from bottom to top respectively. Clearly, $\delta_{\theta_{2}}$ transports $\mu$ through the measure $\sigma:=\delta_{\theta_{2}} \otimes \delta_{0}$ (see Example 4.4), but one has in this situation $\mu_{t}=\delta_{(-\cos t, \sin t)}$ when $t \in[0, \pi / 2)$, and $\mu_{t}=\delta_{(0,1)}$ when $t \geq \pi / 2$, so that the particle stops moving after $\pi / 2$. On the contrary, since $\partial T=0$ what one naturally expects is that the particle never stops moving (because there is "no boundary to reach"). It is not difficult however to assert the existence of such a natural flow, as the following proposition shows. In particular, in the above example the particle under such flow will endlessly rotate along the unit circumference.

Proposition 7.2. Let $\eta$ represent some cycle $T \in \mathcal{M}_{1}(E), \partial T=0$ without cancelation of mass and concentrated over arcwise parameterized curves of unit length (i.e. parameterized with unit speed over $[0,1]$ and stopping definitely at $t=1)$. Then there is a Borel measure $\hat{\eta}$ over $C(\mathbb{R} ; E)$ (equipped with the topology of uniform convergence over bounded intervals) concentrated over $\operatorname{Lip}_{1}(\mathbb{R} ; E)$ such that

(a) $\pi_{\#}^{1} \hat{\eta}=\eta$, where $\pi^{k}: C(\mathbb{R} ; E) \rightarrow C\left(\mathbb{R}^{+} ; E\right)$ is the map defined by $\pi^{k}(\theta)(t):=$ $\theta(t \wedge k), t \in \mathbb{R}^{+}$

(b) $g_{\#}^{ \pm} \hat{\eta}=\hat{\eta}$, where $g^{ \pm}: C(\mathbb{R} ; E) \rightarrow C(\mathbb{R} ; E)$ are the shift maps defined by $g^{ \pm}(\theta)(t):=\theta(t \pm 1)$

(c) for $\hat{\eta}$-a.e. $\theta \in C(\mathbb{R} ; E)$ one has $\theta(\mathbb{R}) \subset \operatorname{supp} T$,

(d) $\pi_{\#}^{k} \hat{\eta}$ represents the current $k T$ without cancelation of mass,

(e) $\hat{\eta}$-a.e. $\theta \in C(\mathbb{R} ; E)$ never stops, namely, it has a.e. constant unit velocity.

If $\mu$ is transportable by $\eta$ through a measure $\sigma=\tilde{\eta} \otimes \lambda_{\theta}$ over $C\left(\mathbb{R}^{+} ; E\right) \times \mathbb{R}^{+}$with $\tilde{\eta}:=q_{\#} \sigma \ll \eta, \tilde{\eta}=\alpha \eta$ for some $\alpha \in L^{1}\left(C\left(\mathbb{R}^{+} ; E\right), \eta\right)$, then $\mu$ is transportable by $\hat{\eta}$ through

$$
\hat{\sigma}:=\left(\left(\alpha \circ \pi^{1}\right) \hat{\eta}\right) \otimes \lambda_{\pi^{1}(\theta)}
$$

If $E$ is a strictly convex Banach space, and $\eta$-a.e. curve is a.e. differentiable, then the respective flow $\hat{\mu}_{t}:=\beta_{t \#} \hat{\sigma}$ satisfies

$$
\frac{\partial \hat{\mu}_{t}}{\partial t}+\operatorname{div} V_{T} \hat{\mu}_{t}=0
$$

in the weak sense and

$$
\dot{\theta}(t)=V_{T}(\theta(t))
$$

for a.e. $t \in \mathbb{R}^{+}$and $\hat{\eta}$-a.e. $\theta \in C\left(\mathbb{R}^{+} ; E\right)$.

Proof. Existence of the measure $\hat{\eta}$ with properties (a)-(c) is just proposition 4.2 from [20], property (d) is remark 4.3 from the same paper, and, finally, (e) follows from (a) and the fact that $\eta$-a.e. $\theta \in C\left(\mathbb{R}^{+}, E\right)$ is parameterized with unit speed over $[0,1]$ stopping definitely at $t=1$. Thus (7.3) follows from (d) combined with Theorem 5.1.

To show that $\mu$ is transportable by $\hat{\eta}$ through $\hat{\sigma}$, we note that $q_{\#} \hat{\sigma}=\left(\alpha \circ \pi^{1}\right) \hat{\eta}$ and recalling (a), we get

$$
\int_{C\left(\mathbb{R}^{+} ; E\right)}\left(\alpha \circ \pi^{1}\right)(\theta) d \hat{\eta}(\theta)=\int_{C\left(\mathbb{R}^{+} ; E\right)} \alpha(\theta) d\left(\pi_{\#}^{1} \hat{\eta}\right)(\theta)=\int_{C\left(\mathbb{R}^{+} ; E\right)} \alpha(\theta) d \eta(\theta) \leq+\infty
$$


because $a \in L^{1}\left(C\left(\mathbb{R}^{+} ; E\right), \eta\right)$. Thus $\alpha \circ \pi^{1} \in L^{1}\left(C\left(\mathbb{R}^{+} ; E\right), \eta\right)$, and hence $q_{\#} \hat{\sigma} \ll \hat{\eta}$. We calculate now $\beta_{0 \#} \hat{\sigma}$. For every $f \in C_{b}(E)$ one has

$$
\begin{aligned}
\int_{E} f(x) d\left(\beta_{0 \# \hat{\sigma})(x)}\right. & =\int_{C\left(\mathbb{R}^{+} ; E\right) \times \mathbb{R}^{+}} f(\theta(s)) d \hat{\sigma}(\theta, s) \\
& =\int_{C\left(\mathbb{R}^{+} ; E\right)} \alpha\left(\pi^{1}(\theta)\right) d \hat{\eta}(\theta) \int_{\mathbb{R}^{+}} f(\theta(s)) d \lambda_{\pi^{1}(\theta)}(s) \\
& =\int_{C\left(\mathbb{R}^{+} ; E\right)} \alpha(\theta) d\left(\pi_{\#}^{1} \hat{\eta}\right)(\theta) \int_{\mathbb{R}^{+}} f(\theta(s)) d \lambda_{\theta}(s),
\end{aligned}
$$

the latter relationship being due to the change of variables and the fact that $\left(\left(\pi^{1}\right)^{-1}(\theta)\right)(s)=\theta(s)$ for $s \in[0,1]$ and that $\lambda_{\theta}$ are concentrated over $[0,1]$. Thus, recalling $(\mathrm{a})$, we get

$$
\begin{aligned}
\int_{E} f(x) d\left(\beta_{0 \#} \hat{\sigma}\right)(x) & =\int_{C\left(\mathbb{R}^{+} ; E\right)} \alpha(\theta) d \eta(\theta) \int_{\mathbb{R}^{+}} f(\theta(s)) d \lambda_{\theta}(s) \\
& =\int_{C\left(\mathbb{R}^{+} ; E\right) \times \mathbb{R}^{+}} f(\theta(s)) d \sigma(\theta, s)=\int_{E} f(x) d\left(\beta_{0 \#} \sigma\right)(x) \\
& =\int_{E} f(x) d \mu(x)
\end{aligned}
$$

because $\mu$ is transportable through $\sigma$. Therefore, $\beta_{0 \#} \hat{\sigma}=\mu$, and thus $\mu$ is transportable also through $\hat{\sigma}$.

It remains to show (7.2). To this aim observe that

$$
\hat{\mu}_{t}=\beta_{t \#} \hat{\sigma}=\beta_{t \#} \hat{\sigma}^{k},
$$

where $k \in \mathbb{N}$ is any natural number satisfying $k \geq\lceil t\rceil+1$, and

$$
\hat{\sigma}^{k}:=\left(\left(\left(\alpha \circ \pi^{1}\right) \circ \pi^{k}\right) \pi_{\#}^{k} \hat{\eta}\right) \otimes \lambda_{\pi^{1}(\theta)}=\left(\left(\alpha \circ \pi^{1}\right) \pi_{\#}^{k} \hat{\eta}\right) \otimes \lambda_{\pi^{1}(\theta)}
$$

(the latter equality being due to the fact that $\pi^{1}\left(\pi^{k}(\theta)\right)=\pi^{1}(\theta)$ for all $\theta \in$ $\left.C\left(\mathbb{R}^{+} ; E\right)\right)$. In particular, this means that $\mu=\hat{\mu}_{0}$ is transportable by $\pi_{\#}^{k} \hat{\eta}$ through $\hat{\sigma}^{k}$. Let now $\tau>0$ be arbitrary and $k:=\lceil t\rceil+1$. Recalling $(\mathrm{d})$ we get $(7.2)$ for $t \in(0, \tau)$ with the help of Theorem $6.1(\mathrm{C})$ (note that $\varphi_{t} \equiv 0$, because $\pi_{\#}^{k} \hat{\eta}$-a.e. curve does not stop definitely). But since $\tau>0$ is arbitrary, we get (7.2) for $t \in \mathbb{R}^{+}$ thus concluding the proof.

\section{ACKNOWLEDGMENTS}

We thank Valentino Magnani for helpful suggestions on how to improve the presentation of Appendix B.

\section{Appendix A. Metric CurRents and measurable Vector fields}

A.1. Metric currents, mass measures and fields of directions. We provide some results on the representation of an arbitrary current $T \in \mathcal{M}_{1}(E)$ in a Banach space $E$ in the form

$$
T(f d \pi)=\int_{E} f(x)\langle V(x), d \pi(x)\rangle d \mu(x)
$$

for some Borel measure $\mu$ and some Borel map $V: E \rightarrow E,\|V\| \in L^{1}(E ; \mu)$, where $\pi \in Q^{1}(E)$. If such a representation exists, we will write (as it is usual in the context of de Rham currents) $T=V \wedge \mu$. Of course such a representation is not unique; for instance $2 V \wedge \mu / 2$ gives the same current. The map $V$ can be viewed as representing the "field of directions" of the current.

Lemma A.1. Let $E$ be a Banach space endowed with the norm $\|\cdot\|$, and $T=$ $V \wedge \mu \in \mathcal{M}_{1}(E)$. Then $m_{T}=\|V(x)\| \mu$. In particular, if $\mu=m_{T}$, then $\|V(x)\|=1$ for $m_{T}$-a.e. $x \in E$. 
Remark A.2. It follows from the above Lemma A.1 that for a given $\mu$ the map $V$ providing the representation (A.1) is unique up to $\mu$-a.e. equality. In fact, if $V \wedge \mu=\tilde{V} \wedge \mu$, then $(V-\tilde{V}) \wedge \mu=0$, and hence one has $\int_{E}\|V-\tilde{V}\| d \mu=0$.

Proof. Since for $\pi \in Q^{1}(E)$ one has $\|d \pi(x)\|^{\prime} \leq \operatorname{Lip} \pi$ for all $x \in E$, the representation (A.1) implies

(A.2) $|T(f d \pi)| \leq \int_{E}|f(x)| \cdot\|d \pi(x)\|^{\prime} \cdot\|V(x)\| d \mu(x) \leq \operatorname{Lip} \pi \int_{E}|f(x)| \cdot\|V(x)\| d \mu(x)$

for $\pi \in Q^{1}(E)$. We may assume without loss of generality $E$ to be separable (if not, just substitute it with the linear span of the closure of a $\sigma$-compact set on which $m_{T}$ is concentrated). Now, as shown in [14], if $\pi \in \operatorname{Lip}_{b}(E)$ with $E$ separable Banach space, then there is a sequence $\pi_{k} \in Q^{1}(E) \cap C_{b}(E)$ with Lip $\pi_{k} \leq \operatorname{Lip} \pi$ converging to $\pi$ pointwise as $k \rightarrow \infty$, so that $\lim _{k} T\left(f d \pi_{k}\right)=T(f d \pi)$. This provides (A.2) for all $\pi \in \operatorname{Lip}_{b}(E)$. At last, if $\pi \in \operatorname{Lip}(E)$, then denoting $\pi_{k}:=(-k) \wedge \pi \vee k$, we have $\pi_{k} \in \operatorname{Lip}_{b}(E)$ with $\operatorname{Lip} \pi_{k} \leq \operatorname{Lip} \pi$, and $\lim _{k} \pi_{k}(x)=\pi(x)$ for every $x \in E$, and therefore $\lim _{k} T\left(f d \pi_{k}\right)=T(f d \pi)$, which means that (A.2) holds for every $f d \pi \in D^{1}(E)$, thus implying $m_{T} \leq\|V\| \mu$ by the definition of the mass measure.

To prove the opposite inequality, note that since $\mu$ is a tight measure, it is concentrated on some separable set $S \subset E$. By lemma 6.10 .16 of [8] the set $V(S)$ is separable (since $V$ is Borel), and thus we may choose a countable dense subset $\left\{\alpha_{j}\right\} \subset V(S)$. For a given $\varepsilon>0$ let

$$
\begin{aligned}
& E_{i}:=\left\{x \in S:\left\|V(x)-\alpha_{i}\right\| \leq \varepsilon\right\} \\
& D_{1}:=E_{1}, \quad D_{i}:=E_{i} \backslash \cup_{j=1}^{i-1} D_{i} .
\end{aligned}
$$

We have that the function $V_{\varepsilon}: E \rightarrow E$ defined by $V_{\varepsilon}(x):=\sum_{i} \alpha_{i} \mathbf{1}_{D_{i}}(x)$ is Borel and $\left\|V(x)-V_{\varepsilon}(x)\right\| \leq \varepsilon$ for $\mu$-a.e. $x \in E$. Let now $a_{j} \in E^{\prime}$ be such that $\left\|a_{j}\right\|^{\prime}=1$ and $\left\langle\alpha_{j}, a_{j}\right\rangle=\left\|\alpha_{j}\right\|$, and set $a_{\varepsilon}(x):=\sum_{i} a_{i} \mathbf{1}_{D_{i}}(x)$, so that $a_{\varepsilon}: E \rightarrow E^{\prime}$ is Borel with $\left\|a_{\varepsilon}(x)\right\|^{\prime}=1$ and $\left\langle V_{\varepsilon}(x), a_{\varepsilon}(x)\right\rangle=\left\|V_{\varepsilon}(x)\right\|$ for $\mu$-a.e. $x \in E$. Let also $\pi_{i}: E \rightarrow \mathbb{R}$ stand for the linear functional $\pi_{i}(x):=\left\langle x, a_{i}\right\rangle$ (so that $\operatorname{Lip} \pi_{i}=1$ ). Then for every Borel $B \subset E$ one gets the estimate

$$
\begin{aligned}
m_{T}(B) & \geq \sum_{i} T\left(\mathbf{1}_{e} \mathbf{1}_{D_{i}} d \pi_{i}\right)=\int_{B}\left\langle V(x), a_{\varepsilon}\right\rangle d \mu(x) \\
& \geq \int_{B}\left\langle V_{\varepsilon}(x), a_{\varepsilon}\right\rangle d \mu(x)-\int_{E}\left\|V(x)-V_{\varepsilon}(x)\right\| \cdot\left\|a_{\varepsilon}(x)\right\|^{\prime} d \mu(x) \\
& \geq \int_{B}\left\|V_{\varepsilon}(x)\right\| d \mu(x)-\varepsilon \mu(E) .
\end{aligned}
$$

Sending $\varepsilon \rightarrow 0^{+}$, we get

$$
m_{T}(B) \geq \int_{B}\|V(x)\| d \mu(x)
$$

which concludes the proof since $B \subset E$ is arbitrary.

Lemma A.3. Let $E$ be a finite-dimensional normed space endowed with the norm $\|\cdot\|$, and $T \in \mathcal{M}_{1}(E)$. Then $T=V_{T} \wedge m_{T}$ for some Borel map $V_{T}: E \rightarrow E$ satisfying $\left\|V_{T}(x)\right\|=1$ for $m_{T}$-a.e. $x \in E$.

Proof. The representation of $T$ in the form (A.1) with $V_{T} \in L^{\infty}\left(E ; m_{T}\right)$ is due to theorem 1.3 from [23] for the case $m_{T} \ll \mathcal{L}^{n}$. To prove it for the general case. let $T_{k} \in \mathcal{M}_{1}(E)$ be such that $T_{k} \rightarrow T, m_{T_{k}} \rightarrow m_{T}$ as $k \rightarrow+\infty$, and $m_{T_{k}} \ll \mathcal{L}^{n}$ for all $k \in \mathbb{N}$ (such a sequence is constructed, say, by convolutions of $T$ with smooth approximate identity, like e.g. in Step 1 of the proof of lemma C.1 from [19]; note that there one only proves that $T_{k} \rightarrow T, \mathbb{M}\left(T_{k}\right) \rightarrow \mathbb{M}(T)$, and then $m_{T_{k}} \rightarrow m_{T}$ is lemma A.3 from [20]). Then $T_{k}=V_{k} \wedge m_{T_{k}}$ for some Borel $V_{k}: E \rightarrow E$ satisfying 
$\left\|V_{k}(x)\right\|=1$ for $m_{T_{k}}$-a.e. $x \in E$. Up to a subsequence (not relabeled) we have that the sequence of vector measures $\left\{V_{k} m_{T_{k}}\right\}$ over $E$ is weakly convergent to some vector measure in $E$ of finite total variation. Denoting the latter limit measure by $V \mu$ for some positive Borel measure $\mu$ and some Borel $V: E \rightarrow \mathbb{R}^{n}$ satisfying $\|V(x)\|=1$ for $\mu$-a.e. $x \in E$, we get for $f d \pi \in D^{1}(E)$ and $\pi \in C^{1}(E) \cap \operatorname{Lip}(E)$ the relationship

$$
T(f d \pi)=\lim _{k} T_{k}(f d \pi)=\int_{E} f(x)\langle V(x), d \pi(x)\rangle d \mu(x),
$$

so that $T=V \wedge \mu$, and hence $m_{T}=\|V\| \mu$ by Lemma A.1. It suffices now to set $V_{T}(x):=V(x) /\|V(x)\|$ (with the convention $0 / 0:=0$ ).

We find it worth providing here a rather particular property of laminated currents regarding the representation (A.1), which, although is not used elsewhere in the paper, is useful to get the clear idea of how different they are from generic metric currents. We will state it in a particular case $E=\mathbb{R}^{n}$ a Euclidean space. Recall the following definition of a tangent space with respect to a finite positive Borel measure $\mu$ over $\mathbb{R}^{n}$ introduced in [9] and further studied in [11]. Denote by $X_{\mu}$ the set of vector valued Borel functions $V: E \rightarrow E$ such that $|V| \in L_{l o c}^{1}(E ; \mu)$ and the distributional divergence $\operatorname{div} V \mu$ defined as

$$
\langle\varphi, \operatorname{div} V \mu\rangle:=\int_{\mathbb{R}^{n}} \nabla \varphi \cdot V d \mu, \quad \varphi \in C_{0}^{\infty}(E),
$$

is a finite signed Radon measure over $E$. Then the tangent space $Q_{\mu}$ to a measure $\mu$ at each point $x \in \mathbb{R}^{n}$ is defined as the $\mu$-essential union of $\left\{V(x): V \in X_{\mu}\right\}$, i.e. $Q_{\mu}: \mathbb{R}^{n} \rightarrow 2^{\mathbb{R}^{n}}$ is the unique $\mu$-measurable closed valued multifunction such that

(i) if $V \in X_{\mu}$, then $V(x) \in Q_{\mu}(x)$ for $\mu$-a.e. $x \in \mathbb{R}^{n}$, and

(ii) it is minimal among all such multifunctions with respect to the inclusion $\mu$-a.e. i.e. if $P: \mathbb{R}^{n} \rightarrow 2^{\mathbb{R}^{n}}$ is another $\mu$-measurable closed valued multifunction such that when $V \in X_{\mu}$, then $V(x) \in P(x)$ for $\mu$-a.e. $x \in \mathbb{R}^{n}$, then necessarily $Q_{\mu}(x) \subset P(x)$ for $\mu$-a.e. $x \in \mathbb{R}^{n}$.

Proposition A.4. If $E=\mathbb{R}^{n}$ is Euclidean space, then for every laminated current $T \in \mathcal{M}_{1}(E)$ the representation $T=V_{T} \wedge m_{T}$ implies $V_{T}(y) \in Q_{m_{T}}(y)$ for $m_{T}$-a.e. $y \in E$.

Remark A.5. Note that the representation $T=V_{T} \wedge m_{T}$ with $V_{T}$ tangent to $m_{T}$ is not true for a generic (not necessarily laminated) current $T$ : in fact, for the current from Remark A.12 one has $Q_{m_{T}}(y)=\{0\}$ for $m_{T}$-a.e. $y \in E=\mathbb{R}$, and hence the existence of such a representation would give $T=0$ which is obviously not the case.

Proof. Let us show that $V_{T}(y) \in Q_{m_{T}}(y)$ for $m_{T}$-a.e. $y \in E$. In fact, this is clearly true if $T$ is normal, since in this case

$$
\left\langle\operatorname{div} V_{T} m_{T}, \varphi\right\rangle=\int_{E} \nabla \varphi \cdot V_{T} d m_{T}=T(1 d \varphi)=\partial T(\varphi), \quad \varphi \in C_{0}^{\infty}(E),
$$

so that $|\langle\operatorname{div} V \mu, \varphi\rangle| \leq \mathbb{M}(\partial T) \cdot\|\varphi\|_{\infty}$, which in other words means $V_{T} \in X_{m_{T}}$. Now passing to the general case of a laminated current $T$, let $B_{k} \subset C\left(\mathbb{R}^{+} ; E\right)$ be an increasing sequence of Borel sets, $B_{k} \nearrow C\left(\mathbb{R}^{+} ; E\right)$, such that the measures $\eta_{k}:=\eta\left\llcorner B_{k}\right.$ be finite. Clearly, one has that $T_{k}:=T_{\eta_{k}}$ are represented by $\eta_{k}$ without cancelation of mass, are normal (because $\eta_{k}$ are finite), and $T_{k} \leq T_{k+1} \leq T$ for every $k \in \mathbb{N}$, and $\mathbb{M}\left(T-T_{k}\right) \rightarrow 0$ as $k \rightarrow \infty$ (because $\ell(\cdot) \eta$ is a finite measure). Thus by Lemma A.6 one has $T_{k}=V \wedge \lambda_{k} m_{T}=\lambda_{k} V \wedge m_{T}$ for some $\lambda_{k} \in L^{\infty}\left(E ; m_{T}\right)$, $0 \leq \lambda_{k} \leq 1$, and, moreover, the sequence of functions $\lambda_{k}$ is pointwise nondecreasing, $\lambda_{k} \nearrow 1$ as $k \rightarrow \infty$. As already proven, one has then $\lambda_{k}(y) V_{T}(y) \in Q_{m_{T}}(y)$ for $m_{T^{-}}$ a.e. $y \in E$. Since for each such $y \in E$ there is a $j \in \mathbb{N}$ such that $\lambda_{k}(y) \geq \lambda_{j}(y)>0$, we have $V_{T}(y) \in Q_{m_{T}}(y)$ as claimed. 
The following auxiliary statement, which is of certain interest by itself, has been used in the above proof.

Lemma A.6. If $E$ is a strictly convex finite-dimensional normed space, $T=V \wedge$ $m_{T} \in \mathcal{M}_{1}(E), S \in \mathcal{M}_{1}(E)$ with $S \leq T$, then there is a $\lambda \in L^{\infty}\left(E ; m_{T}\right)$ with $0 \leq \lambda(x) \leq 1$ for $m_{T}$-a.e. $x \in E$, such that $m_{S}=\lambda m_{T}$ and $S=V \wedge m_{S}=V \wedge \lambda m_{T}$.

Proof. Since $S \leq T$, then $m_{S}+m_{T-S}=m_{T}$, and in particular, $m_{S} \leq m_{T}$ (remark 3.5 from [19]), and hence there is a $\lambda \in L^{\infty}\left(E ; m_{T}\right)$ with $0 \leq \lambda(x) \leq 1$ for $m_{T}$-a.e. $x \in E$, such that $m_{S}=\lambda m_{T}$ and $m_{T-S}=(1-\lambda) m_{T}$. By Lemma A.3 one has $S=V_{S} \wedge \lambda m_{T}=\lambda V_{S} \wedge m_{T}$, with $\left\|V_{S}(x)\right\|=1$ for $\lambda m_{T}$-a.e. $x \in E$, and

$$
T-S=V_{T-S} \wedge(1-\lambda) m_{T}=(1-\lambda) V_{T-S} \wedge m_{T},
$$

with $\left\|V_{T-S}(x)\right\|=1$ for $(1-\lambda) m_{T}$-a.e. $x \in E$. On the other hand,

$$
T-S=\left(V-\lambda V_{S}\right) \wedge m_{T},
$$

so that $V-\lambda V_{S}=(1-\lambda) V_{T-S}$. Thus, for $m_{T}$-a.e. $x \in E$ such that $\lambda(x) \neq 0$ either $\lambda(x)=1$, which implies $V_{S}(x)=V(x)$, or $0<\lambda(x)<1$, which implies

$$
\left\|V(x)-\lambda V_{S}(x)\right\|=1-\lambda(x)=\left\|\left(V(x)\|-\| \lambda(x) V_{S}(x)\right)\right\|,
$$

which is only possible (recalling that $\|V(x)\|=\left\|V_{S}(x)\right\|=1$ ) when $V_{S}(x)=V(x)$, concluding the proof.

Remark A.7. It is easy to observe from the proof of the above Lemma A.6, that its statement is true in a generic metric space $E$, if one know a priori that both $S=V_{S} \wedge m_{S}$ and $T-S=V_{T-S} \wedge m_{T-S}$ for some Borel maps $V_{S}$ and $V_{T-S}$.

A.2. Remarks on measurable vector fields. We mention the following easy properties of measurable vector fields.

(1) (change of measure) If $\psi \ll \mu$, then a $\mu$-vector field is also a $\psi$-vector field (because $L^{\infty}(E ; \mu) \subset L^{\infty}(E ; \psi)$ with continuous embedding; to prove weak continuity one just observes that for each $v \in L^{1}(E ; \psi)$ one has $v \frac{d \psi}{d \mu} \in$ $L^{1}(E ; \mu)$, where $\frac{d \psi}{d \mu}$ stands for the respective Radon-Nikodym derivative).

(2) (locality) If $f(x)=$ const for $\mu$-a.e. $x \in A \subset E$, then $(X f)(x)=0$ for $\mu$-a.e. $x \in A$, if $X$ is a $\mu$-vector field (lemma 13.4 of [16]). In particular, if $f(x)=g(x)$ for $\mu$-a.e. $x \in A$, then $(X f)(x)=(X g)(x)$ for $\mu$-a.e. $x \in A$.

(3) (bounds) One has $|(X f)(x)| \leq C \operatorname{Lip} f$ for $\mu$-a.e. $x \in E$ (with $C>0$ depending on $X$ ). Moreover, thanks to the locality property $X$ can be uniquely extended to the whole $\operatorname{Lip}(E)$ (we will further slightly abuse the notation denoting this extension by the same letter $X$ ) with such a bound (theorem 2.15 from [12]). This extension satisfies weak continuity property in the form $\lim _{k} X f_{k}=X f$ in the weak ${ }^{*}$ sense of $L^{\infty}(E ; \mu)$ whenever $\lim _{k} f_{k}=f$ pointwise and with $\operatorname{Lip} f_{k}$ uniformly bounded. In fact, for a fixed $z \in E$, denoting $f^{r}:=\inf _{B_{r}(z)} f \vee f \wedge \sup _{B_{r}(z)} f$ for an $f \in \operatorname{Lip}(E)$, we get $f=f^{r}$ over $B_{r}(z)$, and hence, for an arbitrary $g \in L^{1}(E ; \mu)$ one has

$$
\begin{aligned}
\int_{E} g X f_{k} d \mu & =\int_{E} g \mathbf{1}_{B_{r}(z)} X f_{k}^{r} d \mu+\int_{E} g \mathbf{1}_{B_{r}(z)^{c}} X f_{k} d \mu, \\
\int_{E} g X f d \mu & =\int_{E} g \mathbf{1}_{B_{r}(z)} X f^{r} d \mu+\int_{E} g \mathbf{1}_{B_{r}(z)^{c}} X f d \mu,
\end{aligned}
$$

so that the claim follows from the estimates

$$
\begin{aligned}
\left|\int_{E} g \mathbf{1}_{B_{r}(z)^{c}} X f_{k} d \mu\right| & \leq \int_{B_{r}(z)^{c}}|g| d \mu \rightarrow 0 \text { as } r \rightarrow+\infty \text { uniformly in } k, \\
\left|\int_{E} g \mathbf{1}_{B_{r}(z)^{c}} X f d \mu\right| & \leq \int_{B_{r}(z)^{c}}|g| d \mu \rightarrow 0 \text { as } r \rightarrow+\infty
\end{aligned}
$$


and from the fact that now $f_{k}^{r}$ are uniformly bounded in $k$ (with a bound depending only on $f(z)$, on the bound on the Lipschitz constants of $f_{k}$, and on $r$ ), and hence $\lim _{k} \int_{E} g \mathbf{1}_{B_{r}(z)} X f_{k}^{r} d \mu=\int_{E} g \mathbf{1}_{B_{r}(z)} X f^{r} d \mu$.

We now show the relationship between measurable vector fields and metric currents.

Proposition A.8. Every $\mu$-vector field $X$ over $E$ defines a metric current $T \in$ $\mathcal{M}_{1}(E)$ by the formula

$$
T(f d \pi):=\int_{E} f X \pi d \mu
$$

with $m_{T} \leq C \mu$. Vice-versa, for every metric current $T \in \mathcal{M}_{1}(E)$ with $m_{T} \leq C \mu$ there is a unique $\mu$-vector field $X$ over $E$ such that (A.3) holds.

Proof. One immediately verifies that if $X$ is a $\mu$-vector field, then $T$ defined by (A.3) is a one-dimensional metric current, and

$$
|T(f d \pi)|:=C \operatorname{Lip} \pi \int_{E}|f| d \mu,
$$

so that $m_{T} \leq C \mu$. On the other hand, if $T \in \mathcal{M}_{1}(E)$, then for every $\pi \in \operatorname{Lip}_{b}(E)$ define $X_{T} \pi \in L^{\infty}\left(E ; m_{T}\right)$ by the relationship

$$
\int_{E} f X_{T} \pi d m_{T}:=T(f d \pi)
$$

for every $f \in L^{1}\left(E ; m_{T}\right)$. It is immediate to verify that $X_{T}$ is a $m_{T}$-derivation. If $\mu \neq m_{T}$, then $m_{T} \ll \mu$ implies by Radon-Nykodym theorem $m_{T}=\alpha \mu$ for some Borel $\alpha: E \rightarrow \mathbb{R}^{+}$. Letting $X \pi:=\alpha X_{T} \pi$, we get

$$
\int_{E} f X \pi d \mu=\int_{E} f \alpha\left(X_{T} \pi\right) d \mu=\int_{E} f X_{T} \pi d m_{T}=T(f d \pi),
$$

and since this is valid for every $f \in L^{1}(E ; \mu)$, hence for every $f \in L^{1}\left(E ; m_{T}\right)$, then $X \pi \in L^{\infty}(E ; \mu)$. The uniqueness of the vector field $X$ follows from the immediate observation that $\int_{E} f X \pi d \mu=0$ for every $f \in L^{1}(E ; \mu)$ means $X \pi=0$ for every $\pi \in \operatorname{Lip}(E)$, hence $X=0$.

Note that if one decides to use the language of measurable vector fields instead of that of currents, then the notion of the boundary of a current has to be substituted by the equivalent one of the divergence of a $\mu$-vector field $X$ defined as a linear continuous functional over $\operatorname{Lip}_{b}(E)$ (equipped with the norm $\|u\|_{\text {Lip }}:=\|u\|_{\infty}+$ Lip $u$ ) by the formula

$$
\left\langle f, \operatorname{div}_{\mu} X\right\rangle:=\int_{E} X f d \mu=T(1 d f)=\partial T(f),
$$

where $f \in \operatorname{Lip}_{b}(E), T \in \mathcal{M}_{1}(E)$, and $\langle\cdot, \cdot\rangle$ stands for the duality between $\operatorname{Lip}_{b}(E)$ and its continuous dual. If $X$ is normal, then in fact the above defined divergence is a signed Radon measure of finite total variation $\left|\operatorname{div}_{\mu} X\right|(E) \leq \mathbb{M}(\partial T)$, and hence the definition (A.4) may be extended to $f \in C_{b}(E)$, and $\langle\cdot, \cdot\rangle$ may be interpreted as the standard duality between $C_{b}(E)$ and the space of signed Radon measure of finite total variation. In the case $E=\mathbb{R}^{n}$ and $\mu$ the Lebesgue measure, when $V: \mathbb{R}^{n} \rightarrow \mathbb{R}^{n}$ is a bounded measurable map (i.e. a finite-dimensional vector field in the classical sense), and $X$ is defined as the classical directional derivative along $V$, i.e. $X f:=V \cdot \nabla f$ for smooth functions $f$, then $\operatorname{div}_{\mu} X$ is identified with the usual distributional divergence of $V$. 
A.3. Representation of currents by measures over curves. The following remarks are worth being made.

Remark A.9. If a nonnegative $\sigma$-finite Borel measure $\eta$ over $C\left(\mathbb{R}^{+} ; E\right)$ is such that

$$
T(\omega)=\int_{C\left(\mathbb{R}^{+} ; E\right)} \llbracket \theta \rrbracket(\omega) d \eta(\theta) \text { for all } \omega \in D^{1}(E),
$$

which we commonly write as $T=T_{\eta}$, then

$$
\mathbb{M}(T) \leq \int_{C\left(\mathbb{R}^{+} ; E\right)} \mathbb{M}(\llbracket \theta \rrbracket) d \eta(\theta) \leq \int_{C\left(\mathbb{R}^{+} ; E\right)} \ell(\theta) d \eta(\theta) .
$$

In fact,

$$
|T(f d \pi)| \leq \int_{C\left(\mathbb{R}^{+} ; E\right)}\left(\operatorname{Lip} \pi \int_{E}|f| d m_{\llbracket \theta \rrbracket}\right) d \eta(\theta),
$$

which gives for a Borel set $B \subset E$ the inequality

$$
m_{T}(B) \leq \int_{C\left(\mathbb{R}^{+} ; E\right)} m_{\llbracket \theta \rrbracket}(B) d \eta(\theta),
$$

and hence in particular (A.6) (plugging $B:=E$ ). The inequality in (A.6) may eventually be strict, as seen even on the trivial example of $\theta_{1}$ being any rectifiable curve parameterized, say, over $[0,1]$, and $\theta_{2}(t):=\theta_{1}(1-t)$, so that $T=0$ satisfies (A.5) with $\eta:=\delta_{\theta_{1}}+\delta_{\theta_{2}}$.

However, if $\eta$ represents $T \in \mathcal{M}_{1}(E)$ without cancelation of mass, then the inequalities in (A.6) become equalities,

$$
\mathbb{M}(T)=\int_{C\left(\mathbb{R}^{+} ; E\right)} \mathbb{M}(\llbracket \theta \rrbracket) d \eta(\theta)=\int_{C\left(\mathbb{R}^{+} ; E\right)} \ell(\theta) d \eta(\theta),
$$

and hence in particular

$$
m_{T}(B)=\int_{C\left(\mathbb{R}^{+} ; E\right)} m_{\llbracket \theta \rrbracket}(B) d \eta(\theta)=\int_{C\left(\mathbb{R}^{+} ; E\right)} d \eta(\theta) \int_{\theta^{-1}(B)}|\dot{\theta}|(t) d t,
$$

for every Borel $B \subset E$ (because the equality holds when $B=E$ ).

An easy consequence of the latter is that $\eta$-a.e. nonconstant $\theta \in C\left(\mathbb{R}^{+} ; E\right)$ belong to the support of $T$, i.e. $\theta \subset \operatorname{supp} T$ (in the sense of traces). In particular, note that then every measure $\eta$ representing $T \in \mathcal{M}_{1}(E)$ without cancelation of mass just under our assumption on tightness of $m_{T}$ is necessarily tight since it is concentrated on a separable space $C\left(\mathbb{R}^{+} ; \operatorname{supp} T\right)$ (recalling that a tight measure is concentrated over its support and the latter is separable).

The following statement combining the results from [19, 20] extending the representation theorems from [21] for currents over a Euclidean space to metric currents, shows is particular that every normal current is laminated (although, of course, there are laminated currents which are not normal).

Proposition A.10. The current $T \in \mathcal{M}_{1}(E)$ is normal, if and only if it is represented by some finite Borel measure $\eta$ over $C\left(\mathbb{R}^{+} ; E\right)$ without cancelation of mass.

Proof. The "only if" part (i.e. the existence of a finite Borel measure $\eta$ representing $T$ without cancelation of mass) is corollary 3.3 of [20], while the "if" part follows from theorem 4.2 from [19].

Remark A.11. The representation of laminated (or even normal) current by some measure over curves is of course not unique: for instance, the current $\llbracket[0,1] \rrbracket$ (in $\mathbb{R}$ ) is represented both by the measure $\delta_{\theta_{1}}+\delta_{\theta_{2}}$ and by $\delta_{\theta_{1} \circ \theta_{2}}$, where $\theta_{1}:=[0,1 / 2]$, $\theta_{2}:=[1 / 2,1]$ and $\theta_{1} \circ \theta_{2}$ stands for the usual composition of curves, i.e. $\theta_{1} \circ \theta_{2}=[0,1]$. However, if $T \in \mathcal{M}_{1}(E)$ is an acyclic normal current, i.e. $C \leq T$ with $\partial C=0$ implies $C=0$, then there is a representation of $T$ by a finite Borel measure $\eta$ over $C\left(\mathbb{R}^{+} ; E\right)$ 
which may be considered canonical, namely $\eta$-a.e. $\theta \in C\left(\mathbb{R}^{+} ; E\right)$ is an injective curve stopping definitely at $t=1$ (hence in particular may be considered without loss of generality to be parameterized over $[0,1])$, and $e_{1 \#} \eta=(\partial T)^{+}, e_{0 \#} \eta=(\partial T)^{-}$, where $e_{i}(\theta):=\theta(i)$ (theorem 5.1 from [19]).

Remark A.12. Not every $T \in \mathcal{M}_{1}(E)$ can be represented by a $\sigma$-finite Borel measure $\eta$ over $C\left(\mathbb{R}^{+} ; E\right)$, and hence the class of laminated currents does not coincide with $\mathcal{M}_{1}(E)$. In the context of classical Whitney flat chains in a Euclidean space the example of a flat chain which is not laminated in the sense of Definition 3.2 has been provided in [10]. We consider here its analogue in the setting of metric currents. Let as in Example $1.1 \mathrm{~K} \subset[0,1]$ be a Cantor set of positive Lebesgue measure (actually every closed totally disconnected set of positive Lebesgue measure will suit) and define the current $T \in \mathcal{M}_{1}(\mathbb{R})$ by setting

$$
T(f d \pi):=\int_{K} f(x) d \pi(x) .
$$

Clearly, $T \neq 0$ and supp $T \subset K$. On the other hand, $T$ cannot be represented by any $\sigma$-finite Borel measure $\eta$ over $C\left(\mathbb{R}^{+} ; E\right)$, because otherwise $\eta$-a.e. $\theta$ would be supported over $K$ by (A.8), and hence would be constant (its trace being a singleton) implying $\llbracket \theta \rrbracket=0$, which would give $T=0$ by (A.5).

Remark A.13. It is worth mentioning that the representation (3.2) does not really depend on the distance in $E$, but rather on the lengths of the curves it generates. In fact, if the distances $d_{1}$ and $d_{2}$ over $E$ have the same geodesic distance $d$ (this means in particular, that the lengths of every absolutely continuous curve $\theta$ for all the these distances coincide, and hence will be denoted by $\ell(\theta)$ ), and $\eta$ represents $T \in \mathcal{M}_{1}\left(E_{1}\right)$ without cancelation of mass, then it also represents the same current but viewed as $T \in \mathcal{M}_{1}\left(E_{2}\right)$ without cancelation of mass, where $E_{1}, E_{2}$ and $E$ stand for $E$ equipped with distances $d_{1}, d_{2}$ and $d$ respectively. In fact,

$$
d_{1}(\operatorname{start}(\theta), \operatorname{end}(\theta)) \leq \ell(\theta)
$$

for every absolutely continuous curve $\theta$, which implies $d \geq d_{1}$, and hence $\mathbb{M}(T) \geq$ $\mathbb{M}_{1}(T)$, where $\mathbb{M}_{1}, \mathbb{M}_{2}$ and $\mathbb{M}$ stand for the masses in $\mathcal{M}_{1}\left(E_{1}\right), \mathcal{M}_{1}\left(E_{2}\right)$ and $\mathcal{M}_{1}(E)$ respectively. But then

$$
\mathbb{M}(T) \leq \int_{C\left(\mathbb{R}^{+} ; E\right)} \ell(\theta) d \eta(\theta)=\mathbb{M}_{1}(T) \leq \mathbb{M}(T),
$$

the first inequality being valid in view of (A.6), and hence all the above inequalities are in fact equalities. Thus, also

$$
\mathbb{M}_{2}(T)=\int_{C\left(\mathbb{R}^{+} ; E\right)} \ell(\theta) d \eta(\theta),
$$

and hence $\eta$ represents $T$ in $\mathcal{M}_{1}\left(E_{2}\right)$ without cancelation of mass.

Remark A.14. If $\eta$ represents $T_{\eta}$ without cancelation of mass and $0 \leq \tilde{\eta} \leq \eta$, then $\tilde{\eta}$ represents $T_{\tilde{\eta}}$ also without cancelation of mass. In fact, one has that $T-T_{\tilde{\eta}}=T_{\eta-\tilde{\eta}}$ and hence by Remark A.9, one gets

$$
\begin{aligned}
\mathbb{M}\left(T_{\tilde{\eta}}\right) & \leq \int_{C\left(\mathbb{R}^{+} ; E\right)} \ell(\theta) d \tilde{\eta}(\theta), \\
\mathbb{M}\left(T_{\eta-\tilde{\eta}}\right) & \leq \int_{C\left(\mathbb{R}^{+} ; E\right)} \ell(\theta) d(\eta-\tilde{\eta})(\theta), \quad \text { so that } \\
\mathbb{M}(T) & \leq \mathbb{M}\left(T_{\tilde{\eta}}\right)+\mathbb{M}\left(T-T_{\tilde{\eta}}\right)=\mathbb{M}\left(T_{\tilde{\eta}}\right)+\mathbb{M}\left(T_{\eta-\tilde{\eta}}\right) \\
& \leq \int_{C\left(\mathbb{R}^{+} ; E\right)} \ell(\theta) d \eta(\theta)=\mathbb{M}(T),
\end{aligned}
$$

which means that the all the inequalities above are in fact equalities. 
We provide also the following lemmata on measures over curves representing some currents without cancelation of mass.

Lemma A.15. Suppose that $\eta$ be a $\sigma$-finite Borel measure over $C\left(\mathbb{R}^{+} ; E\right)$ which represents some laminated current $T \in \mathcal{M}_{1}(E)$ without cancelation of mass, and $\tilde{\eta}$ be another $\sigma$-finite Borel measure over $C\left(\mathbb{R}^{+} ; E\right)$ having $\tilde{\eta} \ll \eta$ and

$$
\int_{C\left(\mathbb{R}^{+} ; E\right)} \ell(\theta) d \tilde{\eta}(\theta)<+\infty .
$$

Then $\tilde{\eta}$ represents $T_{\tilde{\eta}}$ without cancelation of mass.

Proof. One has $\tilde{\eta}=\alpha \eta$ for some $\alpha \in L^{1}\left(C\left(\mathbb{R}^{+} ; E\right), \eta\right), \alpha \geq 0$. The statement is clear when $\alpha$ is a simple (i.e. finite-valued) function. In fact, denoting $\bar{\alpha}$ to be the norm of $\alpha$ in $L^{\infty}\left(C\left(\mathbb{R}^{+} ; E\right), \eta\right)$, we have that $\tilde{\eta} \leq \bar{\alpha} \eta$, while the latter measure represents $T_{\bar{\alpha} \eta}=\bar{\alpha} T_{\eta}$ without cancelation of mass, hence $\tilde{\eta}$ represents $T_{\tilde{\eta}} \leq T_{\bar{\alpha} \eta}$ without cancelation of mass by Remark A.14.

If $\alpha$ is generic, we approximate it as a supremum of simple functions $\alpha_{k}$, converging to $\alpha$ in $L^{1}\left(C\left(\mathbb{R}^{+} ; E\right), \eta\right)$ as $k \rightarrow \infty$. Then for every $\omega=f d \pi \in D^{1}(E)$ one has

and therefore

$$
T_{\alpha_{k} \eta}(\omega)-T_{\alpha \eta}(\omega)=\int_{C\left(\mathbb{R}^{+} ; E\right)} \llbracket \theta \rrbracket(\omega)\left(\alpha(\theta)-\alpha_{k}(\theta)\right) d \eta(\theta),
$$

$$
\mathbb{M}\left(T_{\alpha_{k} \eta}-T_{\alpha \eta}\right) \leq \int_{C\left(\mathbb{R}^{+} ; E\right)} \ell(\theta)\left(\alpha(\theta)-\alpha_{k}(\theta)\right) d \eta(\theta),
$$

the latter integral vanishing as $k \rightarrow \infty$ by Beppo Levi theorem. Hence $T_{\alpha_{k} \eta} \rightarrow$ $T_{\alpha \eta}=T_{\tilde{\eta}}$ in mass as $k \rightarrow \infty$, and in particular

$$
\begin{aligned}
\mathbb{M}\left(T_{\tilde{\eta}}\right)=\lim _{k} \mathbb{M}\left(T_{\alpha_{k} \eta}\right)=\lim _{k} \int_{C\left(\mathbb{R}^{+} ; E\right)} \ell(\theta) \alpha_{k}(\theta) d \eta(\theta) & =\int_{C\left(\mathbb{R}^{+} ; E\right)} \ell(\theta) \alpha(\theta) d \eta(\theta) \\
& =\int_{C\left(\mathbb{R}^{+} ; E\right)} \ell(\theta) d \tilde{\eta}(\theta)
\end{aligned}
$$

again by Beppo Levi theorem, showing the statement.

Lemma A.16. Let $E$ be isometrically embedded into a strictly convex Banach space with Radon-Nikodym property. Under conditions of Lemma A.15 one has then that $T_{\tilde{\eta}}=T\left\llcorner\gamma\right.$ for some $\gamma \in L^{1}\left(E, m_{T}\right)$ (and with $\left.m_{T_{\tilde{\eta}}}=\gamma m_{T}\right)$.

Proof. Since the statement is stable with respect to isometric embeddings of the metric space $E$, we may assume $E$ to be a strictly convex Banach space with RadonNikodym property. The proof will be provided in several steps.

SteP 1. Assume $\tilde{\eta} \leq \eta$. Then $T_{\tilde{\eta}} \leq T_{\eta}=T$. Hence, minding that both $T_{\tilde{\eta}}$ and $T-T_{\tilde{\eta}}=T_{\eta-\tilde{\eta}}$ are laminated currents represented by $\tilde{\eta}$ and $\eta-\tilde{\eta}$ respectively without cancelation of mass by Remark A.14, and hence satisfying the conditions of Remark A.7 in view of Theorem 5.1, we apply the latter Remark A.7 to get the existence of a $\beta \in L^{1}\left(E, m_{T}\right)$, with $0 \leq \beta \leq 1$, such that $S=T\llcorner\beta$.

STEP 2. Assume that $\tilde{\eta}=\alpha \eta$, where $\alpha \in L^{1}\left(C\left(\mathbb{R}^{+} ; E\right), \eta\right)$ is a simple function, i.e. there is a finite partition $P:=\left\{B_{k}\right\}$ of $\eta$-almost all $C\left(\mathbb{R}^{+} ; E\right)$ consisting of Borel subsets $B_{j} \subset C\left(\mathbb{R}^{+} ; E\right)$ such that $\alpha$ is a nonnegative constant $\alpha_{j}$ over each $B_{j}$. Then $T_{\tilde{\eta}}(\omega)=\sum_{k} \alpha_{k} S_{k}(\omega)$, where

$$
S_{k}(\omega):=\int_{C\left(\mathbb{R}^{+} ; E\right)} \llbracket \theta \rrbracket(\omega) d\left(\frac{1}{\alpha_{k}} \tilde{\eta}\left\llcorner B_{k}\right)(\theta)\right.
$$

for every $\omega \in D^{1}(E)$. Applying the result of Step 1 to each $S_{k}$, we get the existence of $\beta_{k} \in L^{1}\left(E, m_{T}\right)$ satisfying $0 \leq \beta_{k} \leq 1$, such that $S_{k}=T\left\llcorner\beta_{k}\right.$, and hence $S=T\llcorner\gamma$ with $\gamma:=\sum_{k} \alpha_{k} \beta_{k}$. 
The following observation regarding this construction is important. Let $\tilde{\eta}^{\prime}=\alpha^{\prime} \eta$, where $\alpha^{\prime} \in L^{1}\left(C\left(\mathbb{R}^{+} ; E\right), \eta\right)$ is a simple function with $\alpha^{\prime} \leq \alpha$. Then $T_{\tilde{\eta}^{\prime}}=T\left\llcorner\gamma^{\prime}\right.$ with $\gamma^{\prime} \leq \gamma$. In fact, there is a finite partition $Q:=\left\{B_{k}\right\}$ of $\eta$-almost all $C\left(\mathbb{R}^{+} ; E\right)$ consisting of Borel subsets $B_{j} \subset C\left(\mathbb{R}^{+} ; E\right)$ such that both $\alpha$ and $\alpha^{\prime}$ are constant over each $B_{j}$. Denote for brevity $\alpha_{j}^{\prime}:=\alpha^{\prime}\left\llcorner B_{j}\right.$, we get $T_{\tilde{\eta}^{\prime}}(\omega)=\sum_{k} \alpha_{k}^{\prime} S_{k}^{\prime}(\omega)$, where

$$
\begin{aligned}
S_{k}^{\prime}(\omega) & :=\int_{C\left(\mathbb{R}^{+} ; E\right)} \llbracket \theta \rrbracket(\omega) d\left(\frac{1}{\alpha_{k}^{\prime}} \tilde{\eta}^{\prime}\left\llcorner B_{k}\right)(\theta)=\int_{C\left(\mathbb{R}^{+} ; E\right)} \llbracket \theta \rrbracket(\omega) d\left(\eta\left\llcorner B_{k}\right)(\theta)\right.\right. \\
& =\int_{C\left(\mathbb{R}^{+} ; E\right)} \llbracket \theta \rrbracket(\omega) d\left(\frac{1}{\alpha_{k}} \tilde{\eta}\left\llcorner B_{k}\right)(\theta)=S_{k}(\omega)=T\left\llcorner\beta_{k}(\omega)\right.\right.
\end{aligned}
$$

for every $\omega \in D^{1}(E)$. Thus, $T_{\tilde{\eta}^{\prime}}=T\left\llcorner\gamma^{\prime}\right.$ with $\gamma:=\sum_{k} \alpha_{k}^{\prime} \beta_{k}$, which implies $\gamma^{\prime} \leq \gamma$ because $\alpha_{k}^{\prime} \leq \alpha_{k}$ for all $k$.

STEP 3. Consider now the general case $\tilde{\eta}=\alpha \eta$ with an arbitrary nonnegative $\alpha \in L^{1}\left(C\left(\mathbb{R}^{+} ; E\right), \eta\right)$. Letting $\alpha_{k} \in L^{1}\left(C\left(\mathbb{R}^{+} ; E\right), \eta\right)$ be a nondecreasing sequence of simple functions with $\alpha_{k} \leq \alpha$ and $\alpha_{k} \nearrow \alpha$ pointwise, we get as in the proof of Lemma A.15 that $T_{\alpha_{k} \eta} \rightarrow T_{\alpha \eta}=T_{\tilde{\eta}}$ in mass as $k \rightarrow \infty$. But according to the result of Step 2, one has $T_{\alpha_{k} \eta}=T\left\llcorner\gamma_{k}\right.$ for some nonnegative $\gamma_{k} \in L^{1}\left(E, m_{T}\right)$, with $\gamma_{k}$ pointwise nondecreasing sequence. Clearly, one has also $T_{\alpha_{k} \eta} \leq T_{\alpha \eta}=T_{\tilde{\eta}}$, so that in particular,

$$
\int_{E} \gamma_{k} d m_{T}=\mathbb{M}\left(T_{\alpha_{k} \eta}\right) \leq \mathbb{M}\left(T_{\tilde{\eta}}\right)<+\infty .
$$

Denoting $\gamma:=\sup _{k} \gamma_{k}$, by Beppo Levi theorem we get $\mathbb{M}\left(T_{\alpha_{k} \eta}\right)=\int_{E} \gamma_{k} d m_{T} \rightarrow$ $\int_{E} \gamma d m_{T}$ as $k \rightarrow+\infty$, but since $\lim _{k} \mathbb{M}\left(T_{\alpha_{k} \eta}\right)=\mathbb{M}\left(T_{\tilde{\eta}}\right)$, we get $\mathbb{M}\left(T_{\tilde{\eta}}\right)=\int_{E} \gamma d m_{T}$. Finally, $\lim _{k} T_{\alpha_{k} \eta}(f d \pi)=\lim _{k} T\left(\gamma_{k} f d \pi\right)=T(\gamma f d \pi)$ for all $f d \pi \in D^{1}(E)$, because $\gamma_{k} f \rightarrow \gamma f$ in $L^{1}\left(E, m_{T}\right)$, and hence $T_{\tilde{\eta}}=T\llcorner\gamma$, proving the claim.

Finally, the lemmata below provide a construction we use in analyzing the transportability condition.

Lemma A.17. Let $\sigma$ be a $\sigma$-finite Borel measure over $C\left(\mathbb{R}^{+} ; E\right) \times \mathbb{R}^{+}$such that $q_{\#} \sigma$ represents some laminated current $T \in \mathcal{M}_{1}(E)$ without cancelation of mass. Then for $S \in \mathcal{M}_{1}(E)$ defined by

$$
S:=\int_{C\left(\mathbb{R}^{+} ; E\right) \times \mathbb{R}^{+}} \llbracket \theta\llcorner[s,+\infty) \rrbracket d \sigma(\theta, s)
$$

one has $S \leq T$ and

$$
\mathbb{M}(S)=\int_{C\left(\mathbb{R}^{+} ; E\right) \times \mathbb{R}^{+}} \ell(\theta\llcorner[s,+\infty)) d \sigma(\theta, s) .
$$

Proof. Letting $R:=\int_{C\left(\mathbb{R}^{+} ; E\right) \times \mathbb{R}^{+}} \llbracket \theta\llcorner[0, s] \rrbracket d \sigma(\theta, s)$, we get

$$
S+R=\int_{C\left(\mathbb{R}^{+} ; E\right) \times \mathbb{R}^{+}} \llbracket \theta \rrbracket d \sigma(\theta, s)=\int_{C\left(\mathbb{R}^{+} ; E\right)} \llbracket \theta \rrbracket d\left(q_{\#} \sigma\right)(\theta)=T,
$$

and

$$
\begin{aligned}
& \mathbb{M}(S) \leq \int_{C\left(\mathbb{R}^{+} ; E\right) \times \mathbb{R}^{+}} \ell(\theta\llcorner[s,+\infty)) d \sigma(\theta, s), \\
& \mathbb{M}(R) \leq \int_{C\left(\mathbb{R}^{+} ; E\right) \times \mathbb{R}^{+}} \ell(\theta\llcorner[0, s]) d \sigma(\theta, s),
\end{aligned}
$$

so that

$$
\mathbb{M}(S)+\mathbb{M}(R) \leq \int_{C\left(\mathbb{R}^{+} ; E\right) \times \mathbb{R}^{+}} \ell(\theta) d \sigma(\theta, s)=\int_{C\left(\mathbb{R}^{+} ; E\right)} \ell(\theta) d\left(q_{\#} \sigma\right)(\theta)=\mathbb{M}(T),
$$

which concludes the proof. 
Lemma A.18. Let $E$ be isometrically embedded in a strictly convex Banach space with Radon-Nikodym property and $T \in \mathcal{M}_{1}(E)$ be a laminated current represented by some $\sigma$-finite Borel measure $\eta$ over $C\left(\mathbb{R}^{+} ; E\right)$ without cancelation of mass, and $\sigma$ be a finite Borel measure over $C\left(\mathbb{R}^{+} ; E\right) \times \mathbb{R}^{+}$such that $q_{\#} \sigma \ll \eta$ and

$$
\int_{C\left(\mathbb{R}^{+} ; E\right)} \ell(\theta) d\left(q_{\#} \sigma\right)(\theta)<+\infty .
$$

Then for an $S \in \mathcal{M}_{1}(E)$ defined by (A.9) one has that $S$ is a normal current, (A.10) is valid and $S=T\left\llcorner\gamma\right.$ for some $\gamma \in L^{1}\left(E, \alpha m_{T}\right)$.

Proof. For the sake of brevity denote $\tilde{\eta}:=q_{\#} \sigma$. By Lemma A.15 this measure represents $T_{\tilde{\eta}}$ without cancelation of mass and by Lemma A.16 one has $T_{\tilde{\eta}}=T\llcorner\alpha$ for some $\alpha \in L^{1}\left(E, m_{T}\right)$. Lemma A.17 says then that $S \leq T_{\tilde{\eta}}$ and is a normal current and (A.10) holds. By Lemma A.6 we get then $S=T_{\tilde{\eta}}\left\llcorner\beta\right.$ for some $\beta \in L^{\infty}\left(E, m_{T}\right)$, with $0 \leq \beta \leq 1$, and to conclude the proof it is enough to set $\gamma:=\alpha \beta$.

\section{Appendix B. Some auxiliary statements}

In this section $E$ stands for an arbitrary Banach space.

B.1. Duality of Banach spaces. For a $v \in E$ we set

$$
v^{\prime}:=\left\{\xi \in E^{\prime}:\langle v, \xi\rangle=\|v\| \cdot\|\xi\|^{\prime}\right\} .
$$

Of course, $v^{\prime} \neq \emptyset$ and $0^{\prime}=E^{\prime}$. Clearly, $v \in v^{\prime \prime}$, where $v^{\prime \prime}:=\left(v^{\prime}\right)^{\prime}$. But if $E$ is strictly convex, then $u \in v^{\prime \prime}, u \in E$ implies $u=\lambda v$ for some $\lambda \in \mathbb{R}^{+}$. In fact, otherwise there is a $\xi \in v^{\prime} \subset E^{\prime}$, with, say, $\|\xi\|^{\prime}=1$ such that $u \in \xi^{\prime}$, and thus $\xi \in u^{\prime}$. The claim follows then from theorem 2 from [15]. Thus for a strictly convex space $E$ one has $v^{\prime \prime} \cap E=\left\{\lambda v: \lambda \in \mathbb{R}^{+}\right\}$.

The following easy statement identifying the equality cases in the Jensen inequality is valid.

Lemma B.1. Let $f \in L^{1}(\Omega, \mu ; E)$, where $(\Omega, \Sigma, \mu)$ is a measure space with some positive $\sigma$-finite measure $\mu$. If

$$
\left\|\int_{\Omega} f(x) d \mu(x)\right\|=\int_{\Omega}\|f(x)\| d \mu(x),
$$

then either $f=0$ or

$$
f(x) \in \frac{\left(\int_{\Omega} f(y) d \mu(y)\right)^{\prime \prime}}{\int_{\Omega}\|f(y)\| d \mu(y)}\|f(x)\|
$$

for $\mu$-a.e. $x \in E$. In particular, if $E$ is strictly convex, then (B.2) implies either $f(x)=0$ or

for $\mu$-a.e. $x \in E$.

$$
f(x)=\frac{\int_{\Omega} f(y) d \mu(y)}{\int_{\Omega}\|f(y)\| d \mu(y)}\|f(x)\|
$$

Proof. We first consider the case when $\mu=\mathbb{P}$ a probability measure. For every $\xi \in\left(\int_{\Omega} f(y) d \mathbb{P}(y)\right)^{\prime}$ one has

$$
\begin{aligned}
\int_{\Omega}\langle f(y), \xi\rangle d \mathbb{P}(y) & =\left\langle\int_{\Omega} f(y) d \mathbb{P}(y), \xi\right\rangle=\left\|\int_{\Omega} f(y) d \mathbb{P}(y)\right\| \cdot\|\xi\|^{\prime} \\
& =\left(\int_{\Omega}\|f(y)\| d \mathbb{P}(y)\right) \cdot\|\xi\|^{\prime},
\end{aligned}
$$

the latter equality being due to (B.2). But since $\langle f(x), \xi\rangle \leq\|f(x)\| \cdot\|\xi\|^{\prime}$ then $\langle f(x), \xi\rangle=\|f(x)\| \cdot\|\xi\|^{\prime}$ for $\mathbb{P}$-a.e. $x \in E$ (since otherwise (B.3) would become a 
strict inequality), which shows

$$
f(x) \in\left(\int_{\Omega} f(y) d \mathbb{P}(y)\right)^{\prime \prime}
$$

for $\mathbb{P}$-a.e. $x \in E$. In the general case assuming $f \neq 0$ and letting

$$
\mathbb{P}:=\frac{\|f\| d \mu}{\int_{\Omega}\|f(y)\| d \mu(y)},
$$

it suffices to refer to apply (B.4) with $f /\|f\|$ instead of $f$.

B.2. Differentiability of curves in Banach spaces. The curve $\theta \in C\left(\mathbb{R}^{+} ; E\right)$ is called weakly (resp. norm) differentiable at $t \in \mathbb{R}^{+}$, if there is a map $\dot{\theta}: \mathbb{R}^{+} \rightarrow E$ satisfying

$$
\lim _{h \rightarrow 0} \frac{1}{h}(\theta(t+h)-\theta(t)-\dot{\theta}(t) h)=0
$$

in the sense of weak (resp. strong, i.e. norm) convergence.

The following more or less folkloric assertions hold true.

Lemma B.2. If a curve $\theta \in C\left(\mathbb{R}^{+} ; E\right)$ is weakly differentiable at a.e. $t \in \mathbb{R}^{+}$, then $\dot{\theta}: \mathbb{R}^{+} \rightarrow E$ is a.e. equal to a Borel function, and, moreover, locally Bochner integrable (resp. Bochner integrable) when $\theta$ is absolutely continuous (resp. rectifiable). Moreover, if $S \subset E$ is some closed separable linear subspace of $E$, and $B \subset C\left(\mathbb{R}^{+} ; S\right)$ is a Borel set of curves weakly differentiable almost everywhere, and $\eta$ is a Borel measure concentrated on $B$, then the map $(\theta, t): B \times \mathbb{R}^{+} \mapsto \dot{\theta}(t)$ is $\eta \otimes \mathcal{L}^{1}$-a.e. equal to a Borel map, and, moreover, is Bochner integrable with respect to $\eta \otimes \mathcal{L}^{1}$ over $B \times K, K \subset \mathbb{R}^{+}$an arbitrary compact set (resp. $B \times \mathbb{R}^{+}$) when $\eta$-a.e. $\theta$ is absolutely continuous and $\theta \mapsto \ell(\theta\llcorner K)$ is $\eta$-integrable (resp. rectifiable and $\theta \mapsto \ell(\theta)$ is $\eta$-integrable $)$.

Proof. For the second part of the statement, we observe that every function $(\theta, t) \in$ $B \times \mathbb{R}^{+} \mapsto\langle\dot{\theta}(t), \psi\rangle \in \mathbb{R}$ for every $\psi \in E^{\prime}$, is is equal for all $\theta \in B$ and a.e. $t \in \mathbb{R}^{+}$ (hence for $\eta \otimes \mathcal{L}^{1}$-a.e. $\left.(\theta, t) \in B \times \mathbb{R}^{+}\right)$to a Borel map $g_{\psi}(\theta, t):=\lim \sup _{k} k\langle\theta(t+$ $1 / k)-\theta(t), \psi\rangle$, and hence the map $(\theta, t): B \times \mathbb{R}^{+} \mapsto \dot{\theta}(t)$ is $\eta \otimes \mathcal{L}^{1}$-weakly measurable. But since for all $\theta \in B$ one has $\dot{\theta}(t) \in S$ whenever defined, i.e. almost everywhere (because $S$ is also weakly closed), and $S$ is separable, then this map is also strongly measurable as claimed. Minding that clearly $\|\dot{\theta}(t)\| \leq|\dot{\theta}|(t)$ for a.e. $t \in \mathbb{R}^{+}$and every $\theta \in B$ (in fact, it is not difficult to prove that the equality holds, but we do not need it), we have also the claimed Bochner integrability under the respective absolute continuity conditions of $\eta$-a.e. $\theta \in B$.

The first part follows from the second one by choosing $B:=\{\theta\}, \eta:=\delta_{\theta}$ the Dirac measure concentrated on $\theta$, and $S \subset E$ any closed separable linear subspace containing $\theta\left(\mathbb{R}^{+}\right)$(which exists because the latter set is separable).

Lemma B.3. If $\theta \in C\left(\mathbb{R}^{+} ; E\right)$ is weakly differentiable a.e. and the weak derivative $\dot{\theta}$ is locally Bochner integrable, then $\theta$ is also norm differentiable a.e. In particular, every absolutely continuous function $\theta \in C\left(\mathbb{R}^{+} ; E\right)$ is weakly differentiable a.e. if and only if it is norm differentiable a.e.

Proof. Observe that

$$
\langle\theta(b)-\theta(a), \psi\rangle=\int_{a}^{b}\langle\dot{\theta}(\tau), \psi\rangle d \tau=\left\langle\int_{a}^{b} \dot{\theta}(\tau) d \tau, \psi\right\rangle
$$


for every $\psi \in E^{\prime}$ and hence $\theta(b)-\theta(a)=\int_{a}^{b} \dot{\theta}(\tau) d \tau$ for every pair $\{a, b\} \in \mathbb{R}^{+}$. Thus

$$
\begin{aligned}
\left\|\frac{\theta(t+s)-\theta(t)}{s}-\dot{\theta}(t)\right\| & =\left\|\frac{1}{s} \int_{t}^{t+s}(\dot{\theta}(\tau)-\dot{\theta}(t)) d \tau\right\| \\
& \leq \frac{1}{|s|} \int_{B_{|s|}(\tau)}\|\dot{\theta}(\tau)-\dot{\theta}(t)\| d \tau \rightarrow 0
\end{aligned}
$$

as $s \rightarrow 0$ whenever $\tau \in \mathbb{R}^{+}$is a Lebesgue point of $\dot{\theta}$ (by proposition 5.3 of [7] a.e. point is so), concluding the proof.

We remark for the sake of completeness, that in case when $E$ is a dual of some Banach space, one can define the notion of weak* differentiability of the curve $\theta: \mathbb{R}^{+} \rightarrow E$ by requiring the limit in (B.5) to be intended in the weak* sense. In this case the first part of Lemma B.3 remains valid (with the obvious modification, i.e. substituting "weak" instead of "weak"), while the second part is not: in fact, as shown in [4], every absolutely continuous curve in a dual of a separable Banach space is weak* differentiable almost everywhere (and the norm of the weak* derivative coincides a.e. with the metric derivative), but clearly might be not norm differentiable unless $E$ has Radon-Nikodym property; therefore, the weak* derivative is not necessarily measurable in the strong sense, and hence first part of Lemma B.2 also fails. The problem is that weak* derivative is just weakly* measurable, but might fail to be separable valued.

\section{REFERENCES}

[1] L. Ambrosio. Lecture notes on optimal transport problems. In Mathematical aspects of evolving interfaces, volume 1812 of Lecture Notes in Mathematics, pages 1-52. Springer-Verlag, 2003.

[2] L. Ambrosio and G. Crippa. Existence, uniqueness, stability and differentiability properties of the flow associated to weakly differentiable vector fields. In Transport equations and multi- $D$ hyperbolic conservation laws, volume 5 of Lect. Notes Unione Mat. Ital., pages 3-57. Springer, Berlin, 2008.

[3] L. Ambrosio and B. Kirchheim. Currents in metric spaces. Acta Math., 185(1):1-80, 2000.

[4] L. Ambrosio and B. Kirchheim. Rectifiable sets in metric and Banach spaces. Math. Ann., $318(3): 527-555,2000$.

[5] L. Ambrosio and D. Trevisan. Well posedness of Lagrangian flows and continuity equations in metric measure spaces. 2014.

[6] M. Beiglböck, A.M.G. Cox, and M. Huesmann. Optimal transport and Skorokhod embedding. Invent. Math., 208:327-400, 2017.

[7] Y. Benyamini and J. Lindenstrauss. Geometric nonlinear functional analysis. Vol. 1, volume 48 of American Mathematical Society Colloquium Publications. American Mathematical Society, Providence, RI, 2000.

[8] V. Bogachev. Measure Theory, vol. I and II. Springer-Verlag, 2006.

[9] G. Bouchitte, G. Buttazzo, and P. Seppecher. Energies with respect to a measure and applications to low-dimensional structures. Calc. Var. Partial Differential Equations, 5(1):37-54, 1997.

[10] L. Brasco and M. Petrache. A continuous model of transportation revisited. Zap. Nauchn. Sem. S.-Peterburg. Otdel. Mat. Inst. Steklov. (POMI), 411(Teoriya Predstavlenii, Dinamicheskie Sistemy, Kombinatornye Metody. XXII):5-37, 240, 2013.

[11] I. Fragalá and C. Mantegazza. On some notions of tangent space to a measure. Proc. Roy. Soc. Edinburgh A, 129(2):331-342, 1999.

[12] J. Gong. Rigidity of derivations on the plane and applications. Illinois J. Math., 56:1109-1147, 2012.

[13] J. Gong. Derivatives and currents on metric (measure) spaces. Real Anal. Exchange, (31st Summer Symposium Conference):217-223, 2007.

[14] V. Goodman. Quasidifferentiable functions on Banach spaces. Proc. Amer. Math. Soc., 30(2):367-370, 1971.

[15] S. Gudder and D. Strawther. Strictly convex normed linear spaces. Proc. Amer. Math. Soc., 59(2):263-267, 1976

[16] J. Heinonen. Nonsmooth calculus. Bull. Amer. Math. Soc. (N.S.), 44(2):163-232, 2007. 
[17] S. Lisini. Characterization of absolutely continuous curves in Wasserstein spaces. Calc. Var. Partial Differential Equations, 28(1):85-120, 2007.

[18] P.-A. Meyer. Convergence faible et compacité des temps d'arrêt d'après Baxter et Chacon. In Séminaire de Probabilités, XII (Univ. Strasbourg, Strasbourg, 1976/1977), volume 649 of Lecture Notes in Math., pages 411-423. Springer, Berlin, 1978.

[19] E. Paolini and E. Stepanov. Decomposition of acyclic normal currents in a metric space. $J$. Funct. Anal., 263(11):3358-3390, 2012.

[20] E. Paolini and E. Stepanov. Structure of metric cycles and normal one-dimensional currents. J. Funct. Anal., 264(3):1269-1295, 2013.

[21] S.K. Smirnov. Decomposition of solenoidal vector charges into elementary solenoids and the structure of normal one-dimensional currents. St. Petersburg Math. J., 5(4):841-867, 1994.

[22] N. Weaver. Lipschitz algebras and derivations. II. Exterior differentiation. J. Funct. Anal., 178(1):64-112, 2000.

[23] M. Williams. Metric currents, differentiable structures, and Carnot groups. Ann. Scuola Norm. Sup. Pisa Cl. Sci. (5), 11(2):259-302, 2012.

Dipartimento di Matematica "U. Dini", Università di Firenze, viale Morgagni 67/A, 50134 Firenze, Italy.

St.Petersburg Branch of the Steklov Mathematical Institute of the Russian Academy of Sciences, Fontanka 27, 191023 St.Petersburg, Russia and Department of Mathematical Physics, Faculty of Mathematics and Mechanics, St. Petersburg State University, Universitetskij Pr. 28, Old Peterhof, 198504 St.Petersburg, Russia and itmO UNIVERSITY, RUSSIA

E-mail address: stepanov.eugene@gmail.com 